\title{
Femtochemistry under scrutiny: Clocking state-resolved channels in the photodissociation of $\mathrm{CH}_{3} \mathrm{I}$ in the $A$-band
}

\author{
Marta L. Murillo-Sánchez ${ }^{1}$, Jesús González-Vázquez ${ }^{2}$, María E. Corrales ${ }^{1}$, Rebeca \\ de Nalda ${ }^{3}$, Emilio Martínez-Núñez ${ }^{4}$, Alberto García-Vela ${ }^{5}$ and Luis Bañares ${ }^{1,6 *}$ \\ ${ }^{1}$ Departamento de Química Física, Facultad de Ciencias Químicas (Unidad \\ Asociada de $I+D+i$ al Consejo Superior de Investigaciones Científicas), \\ Universidad Complutense de Madrid, 28040 Madrid, Spain \\ 2 Departamento de Química and Institute for Advanced Research \\ in Chemical Sciences (IAdChem), Módulo 13, Facultad de Ciencias, \\ Universidad Autónoma de Madrid, 28049 Madrid, Spain \\ ${ }^{3}$ Instituto de Química Física Rocasolano, \\ CSIC, C/ Serrano 119, 28006 Madrid, Spain \\ 4 Departamento de Química Física, \\ Universidade de Santiago de Compostela, \\ 15782 Santiago de Compostela, Spain \\ ${ }^{5}$ Instituto de Física Fundamental, CSIC, \\ C/ Serrano 123, 28006 Madrid, Spain and \\ ${ }^{6}$ Instituto Madrileño de Estudios Avanzados en Nanociencia \\ (IMDEA-Nanoscience), Cantoblanco, 28049 Madrid, Spain
}

(Dated: November 25, 2019)

\footnotetext{
* Corresponding author: lbanares@ucm.es
} 


\begin{abstract}
Clocking of electronically and vibrationally state-resolved channels of the fast photodissociation of $\mathrm{CH}_{3} \mathrm{I}$ in the $A$-band is reexamined in a combined experimental and theoretical study. Experimentally, a femtosecond pump-probe scheme is employed in the modality of resonant probing by resonance enhanced multiphoton ionization (REMPI) of the methyl fragment in different vibrational states and detection through fragment velocity map ion imaging (VMI) as a function of the time delay. We revisit excitation to the center of the $A$-band at $268 \mathrm{~nm}$ and report new results for excitation to the blue of the band center at $243 \mathrm{~nm}$. Theoretically, two approaches have been employed to shed light into the observations: first, a reduced dimensionality 4D non-adiabatic wavepacket calculation using the potential energy surfaces by Xie et al. [J. Phys. Chem. A, 104, 1099 (2000)]; and second, a full dimension 9D trajectory surface-hopping calculation on the same potential energy surfaces, including the quantization of vibrational states of the methyl product. In addition, high level $a b$ initio electronic structure calculations have been carried out to describe the $\mathrm{CH}_{3} 3 p_{z}$ Rydberg state involved in the $(2+1)$ REMPI probing process, as a function of the carbon-iodine (C-I) distance. A general qualitative agreement is obtained between experiment and theory, but the effect of methyl vibrational excitation in the umbrella mode on the clocking times is not well reproduced. The theoretical results reveal that no significant effect on the state-resolved appearance times is exerted by the non-adiabatic crossing through the conical intersection present in the first absorption band. The vibrationally-state resolved clocking times observed experimentally can be rationalized when the $(2+1)$ REMPI probing process is considered. None of the other probing methods applied thus far, i.e multiphoton ionization photoelectron spectroscopy, soft Xray inner-shell photoelectron spectroscopy, VUV single-photon ionization and XUV core-to-valence transient absorption spectroscopy, have been able to provide quantum state-resolved (vibrational) clocking times. More experiments would be needed to disentangle the fine details in the clocking times and dissociation dynamics arising from the detection of specific quantum-states of the molecular fragments.
\end{abstract}




\section{INTRODUCTION}

The idea of timing chemical reactions, in the sense of measuring the time lapses necessary for chemical rearrangement to occur, has been central to Femtochemistry since its early days in the late 1980s, when Ahmed H. Zewail saw the possibility to directly watch the elementary bond cleavage in a molecule using the new capabilities offered by subpicosecond and later femtosecond lasers, $[1-3]$ and applied it for the first time to monitor the C-I bond cleavage of the ICN molecule in real time.[4]

The participation of resonant and non-resonant signals, and how these are a function of the bandwidth and temporal duration of the pulse used for probing a fast reaction were already the subject of Zewail's seminal papers.[2-5] For the so-called clocking experiments, light pulses that are resonant with given electronic transitions of the products of the reaction are employed, either through one photon or multiphoton radiative or non radiative transitions where the observable may be either light (in laser-induced fluorescence experiments)[4], or ionized fragments (in resonance enhanced multiphoton ionization (REMPI) experiments)[6]. In any case, the appearance of a detectable signal indicates that the optical detection window of products is open, and the delay for this to happen is a measurement of the reaction time. [1-3]

The $A$-band of $\mathrm{CH}_{3} \mathrm{I}$ has been one of the prototype systems for ultrafast studies, since dissociation in this band is direct and occurs in the femtosecond regime upon absorption. The reduced number of atoms of the molecule and in contrast, the richness of phenomena involved in dissociation, make it a particularly attractive system. Spectroscopically, the $A$ band is a structureless absorption continuum in the ultraviolet region (220-350 nm), with a maximum at about $260 \mathrm{~nm}$.[7] It corresponds to an $n \rightarrow \sigma^{*}$ transition where a non-bonding $p$ electron of iodine is promoted to the lowest energy available anti-bonding molecular orbital. The heavy iodine atom causes strong spin-orbit coupling, and as a result, the electronic configuration contains five states, named ${ }^{3} Q_{2},{ }^{3} Q_{1},{ }^{3} Q_{0+},{ }^{3} Q_{0-}$ and ${ }^{1} Q_{1}$ in Mulliken notation, all of which are strongly repulsive along the C-I coordinate. Three of these are accessible through dipole allowed transitions: two weak perpendicular transitions to the ${ }^{3} Q_{1}$ and ${ }^{1} Q_{1}$ states and a strong parallel transition to the ${ }^{3} Q_{0+}$ state (thus the ${ }^{3} Q_{0+}$ state will be subsequently called $\left.{ }^{3} Q_{0}\right)$. In fact, in the maximum of the absorption band, absorption of light takes place almost exclusively upon excitation through a strong parallel transition from the 
ground electronic state to the ${ }^{3} Q_{0}$ electronic state. Excitation of this surface adiabatically leads to the formation of the iodine atom in its spin-orbit excited state, $\mathrm{I}^{*}\left({ }^{2} P_{1 / 2}\right)$, whereas all the other surfaces correlate with ground state iodine atoms, $\mathrm{I}\left({ }^{2} P_{3 / 2}\right)$. The presence of non-adiabatic couplings between the surfaces - the most relevant of which is the conical intersection between the ${ }^{3} Q_{0}$ and the ${ }^{1} Q_{1}$ states - complicates the origin of iodine atoms, which can in principle be produced via both adiabatically and non-adiabatically. In first approximation, it is possible to conceive this process as an essentially one-dimensional problem, given the lightness of the hydrogen atoms attached to the carbon atom, and the fact that dissociation is direct and fast along the C-I bond. However, some of the other degrees of freedom play an essential role in the process, in aspects as fundamental as the non-adiabatic crossing that leads to the formation of iodine atoms, which is only possible when the $C_{3 v}$ symmetry is broken[8] (which corresponds to $\mathrm{H}_{3} \mathrm{C}$-I bending during dissociation). It is also important to recall that the $\mathrm{CH}_{3}$ moiety changes from pyramidal to planar geometry, which causes important umbrella mode excitation.

Extensive experimental work has been devoted to the study of this fundamental process, both in asymptotic (using nanosecond pulsed lasers) [9-13] and time-resolved (using femtosecond pulsed lasers) contributions [14-19]. Eppink and Parker applied their newly discovered velocity map imaging technique to the study of this process $[10,11]$ across the full $A$-band, and the blue [12] and red [13] edges of the absorption band have been explored with slice imaging and nanosecond pulsed lasers.

Also, numerous theoretical works have dealt with this problem $[8,15,16,20-28]$ using various approaches including a full-dimensional (9D) quantum dynamics study [27]. Despite the intense efforts, controversy or discrepancy with experiment persists on aspects like the description of the absorption spectrum, the relevance of the absorption strength to the three dipole-allowed ${ }^{3} Q_{1},{ }^{1} Q_{1}$ and ${ }^{3} Q_{0}$ states or the $\mathrm{CH}_{3}$-vibrationally-selected quantum yields.

The comparison of wavepacket calculations performed for the center of the $A$-band in $\mathrm{CH}_{3} \mathrm{I}$ with the results of femtosecond velocity map imaging experiments were reported a few years ago $[15,16]$. The $\mathrm{CH}_{3} \mathrm{I}$ photodissociation dynamics was studied using a wavepacket model including four degrees (4D) of freedom [16], namely the C-I dissociation coordinate, the $\mathrm{I}-\mathrm{CH}_{3}$ bending mode, the $\mathrm{CH}_{3}$ umbrella mode, and the $\mathrm{C}-\mathrm{H}$ symmetric stretch mode. The theoretical reaction times are compared with those obtained in femtosecond velocity map imaging experiments. The model reproduces very well the experimental reaction times 
for the $\mathrm{CH}_{3}\left(\nu_{1}, \nu_{2}\right)+\mathrm{I}^{*}\left({ }^{2} P_{1 / 2}\right)$ dissociation channels with $\nu_{1}=0$ and $\nu_{2}=0,1,2$, and also for the channel $\mathrm{CH}_{3}\left(\nu_{1}=0, \nu_{2}=0\right)+\mathrm{I}\left({ }^{2} P_{3 / 2}\right)$. The model fails, however, to predict the experimental clocking times for the $\mathrm{CH}_{3}\left(\nu_{1}, \nu_{2}\right)+\mathrm{I}\left({ }^{2} P_{3 / 2}\right)$ channels with $\left(\nu_{1}, \nu_{2}\right)=(0,1),(0,2)$, and $(1,0)$, i.e., when the $\mathrm{CH}_{3}$ fragment produced along with spin-orbit ground state I atoms is vibrationally excited. Different explanations were given for the disagreement, but the differences have not been assessed definitely.

Meanwhile, experiments on the photodissociation of $\mathrm{CH}_{3} \mathrm{I}$ in the $A$-band using different spectroscopic techniques for probing the fragments in a time-resolved fashion and even following the time evolution of the transition-state region have been carried out in the last few years [29-33]. Attar et al. [29] were the first to measure the transition-state region in the $A$-band photodissociation of $\mathrm{CH}_{3} \mathrm{I}$ at $266 \mathrm{~nm}$ using femtosecond extreme ultraviolet (XUV) transient absorption spectroscopy by real-time evolution of core-to-valence transitions near the iodine N-edge at 45-60 eV. Core-to-valence electronic states decay during the C-I bond cleavage simultaneously to the $\mathrm{I}\left({ }^{2} P_{3 / 2}\right)$ and $\mathrm{I}^{*}\left({ }^{2} P_{1 / 2}\right)$ fragments resonant appearance. They found that the transition-state resonances reach a maximum at $\sim 40$ fs and decay to complete C-I dissociation in $\sim 90$ fs. Later, Drescher et al. [30] reported similar experiments as those of Ref. [29], but they observed a continuous shift in energy of the emerging atomic absorption lines which were attributed to relaxation of the valence shell vacancy that occur during the first few femtoseconds of the C-I bond dissociation.

Baumann et al. [31] performed single-shot UV/VUV pump-probe experiments to study the valence state photodissociation of $\mathrm{CH}_{3} \mathrm{I}$ after excitation at $268 \mathrm{~nm}$ and probing by one or two-photon ionization at $161 \mathrm{~nm}$ with a sub-10 fs time resolution. The reversed order of the UV and VUV femtosecond laser pulses allowed them to study the dissociation from the $6 p\left({ }^{2} E_{3 / 2}\right)$ Rydberg state after excitation at $161 \mathrm{~nm}$ and probing by multiphoton ionization at $268 \mathrm{~nm}$. They attributed time constants of $98.2 \pm 1.9 \mathrm{fs}$ and $28.5 \pm 2.8$ fs for the biexponential decay of the $\mathrm{CH}_{3} \mathrm{I}$ parent ion of the $3 Q_{0}$ and ${ }^{1} Q_{1}$ states, respectively. The ${ }^{3} Q_{0}$ time constant was directly related to the C-I bond cleavage along the reaction coordinate up to the asymptotic region of the potential energy surface, whereas the ${ }^{1} Q_{1}$ time constant was attributed to the duration of the ${ }^{1} Q_{1} \rightarrow \tilde{X}^{1} E_{3 / 2}$ ionization window considering the restrictions imposed by the VUV probing wavelength $(161 \mathrm{~nm})$. Mechanisms were proposed to account for the time constants observed for the fragment ions $\mathrm{I}^{+}$and $\mathrm{CH}_{3}^{+}[31]$.

Inner-shell photoelectron spectroscopy was employed by Brausse et al. [32] to time- 
resolve the ultrafast photodissociation of $\mathrm{CH}_{3} \mathrm{I}$ at $272 \mathrm{~nm}$ in the form of structural and electronic transformations, using $\sim 120$ fs soft-X-ray pulses centered at $11.6 \mathrm{~nm}$ as a probe, to ionize above the iodine $4 d$ edge, and photoelectron-photoion coincidences. The observed reduction in intensity of the iodine $4 d$ peak in the photoelectron spectrum together with the appearance of a contribution attributed to ionization of iodine atoms was assigned to the evolution from the bound molecule to the isolated fragment atom, although the time resolution of the experiment was not enough to fully resolve the process.

Finally, Warne et al. [33] studied the time-resolved pump-probe photodissociation of $\mathrm{CH}_{3} \mathrm{I}$ on the red $(269 \mathrm{~nm})$ and blue $(255 \mathrm{~nm})$ parts around the center of the $A$-band by UV (395 nm) multiphoton ionization photoelectron spectroscopy. Surprisingly, they observed different dynamics at the two excitation wavelengths with significant differences in the measured reaction times and dynamic structure, which were attributed to a significant contribution of the ${ }^{1} Q_{1}$ state at the excitation wavelength of $255 \mathrm{~nm}$, i.e. more complex structure in the photoelectron spectrum and increased appearance time, indicative of a more complex reaction pathway on the ${ }^{1} Q_{1}$ potential energy surface.

In this work, we present a combined experimental and theoretical study where excitation slightly to the red $(268 \mathrm{~nm})$ and to the blue $\left(243 \mathrm{~nm}\right.$ ) of the $A$-band of $\mathrm{CH}_{3} \mathrm{I}$ (maximum at $260 \mathrm{~nm}$ ) is explored in detail, providing new insights into the unresolved issues concerning the quantum state (vibrational and electronic) resolved clocking times for this photodissociation process. It is, to our knowledge, the first time where time-resolved measurements are carried out nearer to the blue edge of the band, which is of particular interest due to the possible contribution of more than one excited state [13]. Furthermore, improvements in the analysis of the velocity map images as a function of time delay has permitted a more detailed determination of anisotropies in the spatial distribution of the methyl fragments and reaction (clocking) times. The findings are rationalized with the help of two combined theoretical approaches, a 4D wave packet calculation and a full dimension (9D) trajectory surface hopping calculation with quantization of the final vibrational states of the methyl fragment, that help to unravel the complex dynamics at play in the fast photodissociation of this prototypical polyatomic molecule. 


\section{METHODOLOGIES}

\section{A. Experimental}

The femtosecond laser system, a chirped pulse amplified Ti:sapphire, delivers $3.5 \mathrm{~mJ}$ pulses of $50 \mathrm{fs}$ duration at a repetition rate of $1 \mathrm{kHz}$ with a tunable central wavelength of about $804 \mathrm{~nm}$. The output of the amplifier is split in two arms to provide the pump and probe pulses.

In the pump arm, UV femtosecond laser pulses are synthesized for the excitation of $\mathrm{CH}_{3} \mathrm{I}$ in its first absorption band ( $A$-band). For this purpose, the fundamental output $(\sim 1 \mathrm{~mJ})$ is used to pump a two-stage femtosecond automated optical parametric amplifier (TOPAS). The output signal is then twice frequency doubled in two BBO crystals in order to obtain tunable radiation at the blue side of the $A$-band, at $243 \mathrm{~nm}$, with $7-9 \mu \mathrm{J}$ per pulse. Alternatively, for excitation at the center of the $A$-band at $268 \mathrm{~nm}$, the fundamental output is frequency tripled with BBO crystals.

The probe arm is designed to provide probe radiation to resonantly ionize the $\mathrm{CH}_{3}$ products by $(2+1)$ REMPI through the $3 p_{z}$ Rydberg state. The fundamental beam is used to pump an optical parametric amplifier (OPA), where signal and idler pulses are generated in a BBO crystal. The signal pulse is later frequency quadrupled to constitute the probe beam providing tuneable pulses in the 325-334 nm region with pulse energies typically around 6-7 $\mu \mathrm{J}$ and with a full width at half maximum (FWHM) bandwidth of $\approx 3 \mathrm{~nm}$. Tuning these pulses in the above mentioned range allows $(2+1)$ REMPI detection of $\mathrm{CH}_{3}$ radicals in different vibrational states: vibrationless state $\nu=0$ at $333.5 \mathrm{~nm}$, symmetric stretch excited $\nu_{1}=1$ at $333.9 \mathrm{~nm}$, umbrella-mode excited $\nu_{2}=1$ and $\nu_{2}=2$ at $329.4 \mathrm{~nm}$ and $325.8 \mathrm{~nm}$, respectively, and accidentally the corresponding combination bands $\left(\nu_{1}, \nu_{2}\right)=(1,1)$ and $\left(\nu_{1}, \nu_{2}\right)=(1,2)$.

The linear polarization of the pump and probe laser beams is set up horizontal by means of half-wave plates and the propagation conditions are controlled through adjustable telescopes. Pump and probe beams are propagated collinearly and finally focused with a $25 \mathrm{~cm}$ focal length lens into a vacuum chamber where they interact with a pulsed molecular beam.

The supersonic molecular beam is generated by expansion of $\mathrm{CH}_{3} \mathrm{I}$ at $0^{\circ} \mathrm{C}$ in helium at a backing pressure of 1.5-2 bars, through a $0.5 \mathrm{~mm}$ diameter nozzle, $1 \mathrm{kHz}$ homemade cantilever piezoelectric pulsed valve [15]. A 1 mm diameter skimmer separates the expansion 
and ionization chambers, where the molecular beam interacts with the laser pulses. Clustering conditions in the beam are avoided by keeping low enough pressure and working on the initial part of the molecular beam pulse.

The delay between the pump and probe pulses is controlled by a motorized delay stage placed at the probe laser arm that allows time steps as low as $1 \mathrm{fs}$. The instrument temporal response time, considered as the temporal cross correlation of the pump and probe pulses, is measured through multiphoton ionization of Xe, obtaining typical values of $150 \mathrm{fs}$.

After interaction with the laser pulses, the ions formed are extracted in the vertical direction by a set of electrostatic lenses working in velocity mapping configuration [34]; typical repeller voltages are $5200 \mathrm{~V}$, with optimum velocity mapping conditions found for $V_{\text {extractor }} / V_{\text {repeller }}=0.76$. The field-free time-of-flight $(\mathrm{TOF})$ region is $50 \mathrm{~cm}$ long. The detector is composed of a dual microchannel plate (MCP) in Chevron configuration, coupled to a phosphor screen. Mass selection is achieved through gating the gain in the front MCP plate. The images thus generated on the phosphor screen are recorded with a Peltier-cooled 12 bit charge-coupled device camera.

The energy calibration of the apparatus is done by measuring the $\mathrm{CH}_{3}^{+}$image upon 268 $\mathrm{nm}$ photodissociation of $\mathrm{CH}_{3} \mathrm{I}$ and resonant multiphoton ionization of $\mathrm{CH}_{3}(\nu=0)$, and using the known kinetic energy release (KER) of the $\mathrm{CH}_{3}(\nu=0)+\mathrm{I}^{*}\left({ }^{2} P_{1 / 2}\right)$ and $\mathrm{CH}_{3}(\nu=0)+$ $\mathrm{I}\left({ }^{2} P_{3 / 2}\right)$ channels $[11,15]$.

For the realization of a clocking experiment, once the signal is optimized and the time overlap is found, several sets of images are acquired as the pump-probe delay is scanned over a given time range. Each image is the result of accumulating over 800 laser shots. Finally, the equivalent images of all the sets are added together. Images are Abel inverted using the polar basis set expansion (pBasex) method [35] and a multidimensional fitting model is used for the analysis of the data [36]. This model will be described in some detail below.

For the clocking measurements, two different methods have been employed for the determination of time zero, i.e. the temporal overlap between the pump and probe lasers. Firstly, typical in situ measurements of the transient from multiphoton ionization of xenon were performed allowing also the pump-probe cross-correlation function (120 fs) to be measured. Secondly, a low-energy contribution appearing in the centre of all images, which is attributed to multiphoton dissociative ionization processes, has also been used. Both transients are fitted to Gaussian functions and they coincide with each other within \pm 20 fs. 


\section{B. 4D wavepacket calculations}

The $\mathrm{CH}_{3} \mathrm{I}$ molecule is an ideal prototype to develop theoretical models to describe photodissociation processes $[37,38]$. In this work, the $\mathrm{CH}_{3} \mathrm{I}$ system is represented by considering four degrees of freedom $(R, r, \alpha, \theta)$, where the dissociation coordinate $R$ is the distance between I and the $\mathrm{CH}_{3}$ center-of-mass, $r$ is the symmetric $\mathrm{C}-\mathrm{H}$ bond distance representing the $\mathrm{CH}_{3}$ symmetric stretch mode ( $\nu_{1}$ mode), $\alpha$ is the umbrella bending angle of the $\mathrm{CH}_{3}$ group ( $\nu_{2}$ mode), and $\theta$ is the in-plane $\mathrm{H}_{3}$ - $\mathrm{C}-\mathrm{I}$ bending angle ( $\nu_{6}$ mode) between the $\mathrm{C}-\mathrm{I}$ axis and the axis perpendicular to the plane containing the three hydrogen atoms. [16] Wavepacket simulations including five degrees of freedom, namely the current four modes plus the outof-plane bending mode, have shown that this latter mode plays a small role in the $\mathrm{CH}_{3} \mathrm{I}$ photodissociation dynamics and can be ignored [23], and this is why it is neglected in the present model. Furthermore, full-dimensional, 9D calculations carried out using the multiconfiguration time-dependent Hartree (MCTDH) scheme have been reported [27], and they show that the 5D and 9D descriptions lead to rather similar results. Thus, it is expected that the four modes considered in the current model are able to reliably describe the main features of the $\mathrm{CH}_{3} \mathrm{I}$ non-adiabatic photodissociation dynamics in the $A$-band. It is also noted that the present model allows one to represent the dynamics of the $\mathrm{CH}_{3}$ symmetric stretch mode $\nu_{1}$, and to describe dissociation channels producing $\mathrm{CH}_{3}$ fragments in the $\nu_{1}=0$ and 1 states, which are probed experimentally.

At the $\lambda=243$ and $268 \mathrm{~nm}$ excitation wavelengths used in this work the intensity of the ${ }^{3} Q_{1} \leftarrow \tilde{X}{ }^{1} A_{1}$ transition is very small, as shown by the experimental data and the theoretical $A$-band spectrum [12]. Thus, in the present model the ${ }^{3} Q_{1} \leftarrow \tilde{X}{ }^{1} A_{1}$ transition is neglected, and only the ${ }^{3} Q_{0} \leftarrow \tilde{X}{ }^{1} A_{1}$ and ${ }^{1} Q_{1} \leftarrow \tilde{X}{ }^{1} A_{1}$ transitions are considered. A detailed description of the representation of the potential energy surfaces of the different electronic states involved in the photodissociation process and of the transition dipole moment functions for the ${ }^{3} Q_{0} \leftarrow \tilde{X}{ }^{1} A_{1}$ and ${ }^{1} Q_{1} \leftarrow \tilde{X}^{1} A_{1}$ transitions has been given elsewhere [8, 12, 15, 16, 24-26]. The Hamiltonian, the wave packet method used to simulate the photodissociation of $\mathrm{CH}_{3} \mathrm{I}$, and the procedure applied to obtain the observable quantities of interest have been described in detail previously [16].

There is, however, a significant difference between the present calculations and those of the previous work [16]. In the earlier work all the calculated magnitudes were averaged 
over the spectral bandwidth of both the pump and probe pulses used in the experiment [16]. Taking into account the influence of these experimental conditions in the simulations led to a more favorable comparison between the experimental and theoretical results. As previously described [16], the experimental detection energy window originated by the probe pulse bandwidth was simulated in the calculations by projecting out the wave packet at different asymptotic $\mathrm{CH}_{3}-\mathrm{I}$ distances $R_{c}$, and averaging over the results obtained. Such an average over the probe pulse spectral bandwidth has been supressed in the present simulations (albeit the average over the pump pulse bandwidth is still performed), and currently the magnitudes of interest, and particularly the appearance times, are calculated by projecting out the wave packet only at the single asymptotic distance $R_{c}=13$ bohr $(6.88 \AA)$, the same one that is used in the surface-hopping trajectory simulations (see below). This asymptotic distance has been chosen considering that the interaction between the heavy iodine atom and the electronic states of the methyl fragment, specially those with Rydberg character, is negligible as reflected in the $a b$ initio calculations of the Rydberg states of the methyl fragment as a function of the C-I distance (see below), and, thus, the methyl fragment can be considered as a purely free fragment. Although choosing an asymptotic distance is always arbitrary, the aim here is to use similar conditions in the calculation of the observable quantities in both the wavepacket and trajectory treatments, in order to make the corresponding results as comparable as possible and not influenced by the probing process. This way we can observe subtle effects on the clocking times due, for instance, to the non-adiabatic crossing through the conical intersection between the ${ }^{3} Q_{0}$ and ${ }^{1} Q_{1}$ potential energy surfaces, to the methyl vibrational mode selectivity or to the different available energy for the bond cleavage depending on the final vibrational and electronic states of the fragments.

It is well known that the transition dipole moment functions based on ab initio data used to describe the ${ }^{1} Q_{1} \leftarrow \tilde{X}{ }^{1} A_{1}$ transition clearly overestimates the absorption to this state in comparison with experimental determinations $[12,16]$. This is specially relevant in the present work, since we have studied the photodissociation of $\mathrm{CH}_{3} \mathrm{I}$ in the blue part of the $A$-band at $243 \mathrm{~nm}$, where according to the theoretical absorption spectrum, the ${ }^{1} Q_{1}$ state will absorb substantially in an amount comparable to the absorption to the ${ }^{3} Q_{0}$ state [12] (see Figure 1 below). 


\section{9D surface-hopping trajectory calculations}

The laser induced non-adiabatic dynamics has been simulated in full dimensionality by using semiclassical methodology. In this methodology, the nuclear degrees of freedom follow the classical Newton equations and the electronic dynamics is described by the timedependent Schrödinger equation. We use the SHARC method [39] in combination with the full dimension potential energy surfaces reported by Amatatsu et al. [8, 24].

First, the minimum geometry and harmonic frequencies are obtained using perturbation theory (CASPT2) from a state average complete active space calculation including the first 3 singlet states using the $6-31 \mathrm{G}$ basis set and a pseudopotential for the iodine atom. The lone pairs of the iodine atom and the I-C bonding and antibonding orbitals were included in the active space SA3-CASPT2 (6,4). These calculations have been performed with the MOLPRO package [40].

Using this geometry, a set of 2 million initial conditions were created using a Wigner distribution that were employed to evaluate the semiclassical spectrum depicted in Figure 1, considering both transitions to the ${ }^{3} Q_{0}$ and to the ${ }^{1} Q_{1}$ states according to the model from Ref. [12]. Using this spectrum, a stochastic sampling of initial conditions was performed in the energy regions between 5.08 and $5.12 \mathrm{eV}$ (for excitation at $243 \mathrm{~nm}$ ) and between 4.61 and $4.65 \mathrm{eV}$ (for excitation at $268 \mathrm{~nm}$ ), selecting 22850 and 74140 initial conditions, respectively. Trajectories using these sets of initial conditions were run with the SHARC code [39] during 300 fs using the full dimension (9D) analytical potential energy surfaces described by Amatatsu et al. [8] and corrected by Xie et al. [24]. During each trajectory calculation, the adiabatic surfaces were obtained by the diagonalization of the electronic Hamiltonian (potential and diabatic couplings) where the gradient was evaluated to follow the classical movement. On the other hand, the dynamics of the electronic wavefunction was followed using a Runge-Kutta algorithm of fourth order. An stochastic algorithm based on the density [41] was employed to decide the electronic state where the potential runs using a surface hopping algorithm.

Finally, the fragments kinetic energy and the appearance time were recorded for every trajectory. The appearance time was obtained employing the same methodology described in the wave packet calculations, i.e. for every trajectory, the time where the distance between the $\mathrm{I}$ atom and the $\mathrm{CH}_{3}$ moeity is 13 bohr $(6.88 \AA)$ was recorded. 




FIG. 1: Semiclassical absorption spectrum corresponding to the ${ }^{3} Q_{0} \leftarrow \tilde{X}{ }^{1} A_{1}$ (parallel) and the ${ }^{1} Q_{1} \leftarrow \tilde{X}^{1} A_{1}$ (perpendicular) transitions calculated with 2 million initial conditions using a Wigner distribution. The vertical bars represent the selected trajectories for excitation at 243 $\mathrm{nm}$ (orange) and $268 \mathrm{~nm}$ (violet) considering the femtosecond laser pulse bandwidth. Notice that the contribution from the perpendicular transition is clearly overestimated in comparison with experimental determinations [7] when using the transition dipole moments based on the ab initio data of Alekseyev et al. [26] as modeled in Ref. [12].

To obtain the vibrational quantum numbers of the methyl fragment, the normal mode analysis (NMA) approach of Corchado and Espinosa-García is employed [42]. Briefly, in the NMA method the vibrational energy content of each vibrational mode $i, E_{i}$, is the sum of a kinetic, $E_{k i n, i}$, and a potential energy $E_{p o t, i}$, which are computed from the projection onto the normal mode space of the momenta and unscaled Cartesian coordinates of the fragment, respectively. Corchado and Espinosa-García have also incorporated an anharmonic correction and Coriolis coupling to the NMA approach [42]. The method has been tested against the Fast Fourier Transform (FFT) method [43-46] for polyatomic molecules and the Einstein-Brillouin-Keller (EBK) [47] method for diatomics. NMA has also been recently employed to study the vibrational energy distributions of hydrogen cyanide resulting in the 
photolysis of vinyl cyanide[48] and methyl cyanoformate.[49]

\section{Ab initio calculations of the $\mathrm{CH}_{3}$ Rydberg states}

In order to evaluate the influence of the proximity of the iodine atom to the energy of the $\mathrm{CH}_{3}$ Rydberg states involved in the $(2+1)$ REMPI probing process used in this work, the relevant Rydberg states have been calculated as a function of the C-I distance with restricted active space methods. The basis set was constructed by using a complete ANORCC basis set [50] in the $\mathrm{CH}_{3}$ radical, whereas in the I atom a triple zeta contraction scheme was employed. In this way, it is possible to describe very diffuse orbitals in the $\mathrm{CH}_{3}$ radical, maintaining a reasonable computational cost. The active space was selected in the dissociating limit including the three lone pairs of the I atom together with the lone electron, and the Rydberg orbitals, $3 s$ and $3 p_{z}$, of the $\mathrm{CH}_{3}$ fragment. In order to reduce the size of the configuration space, a restricted active space formalism was followed. In our case, we divide the space into two parts: the RAS2 space containing the I orbitals and the lone pair in the $\mathrm{CH}_{3}$, where all excitations are allowed, and the Rydberg orbitals in the RAS3 space, where only single excitations were possible. Two state-averaged calculations were performed for 16 singlets and 15 triplets states. Relativistic effects were considered by using a Douglas-Kroll Hamiltonian [51] and the spin-orbit coupling between the singlet and triplet manifolds was computed in the frame of the Atomic Mean Field Approximation (AMFI) [52], obtaining a total of 61 electronic states. Finally, a one dimensional grid was calculated by freezing the $\mathrm{CH}_{3}$ moeity in its CASPT2 minimum and the I atom approaching starting at $10 \AA$ until 4 A. These calculations were performed with the OpenMolcas code [53].

In order to calculate the adiabatic potential energy curves for the umbrella mode, a 2D grid including the $R$ and $r$ distances, representing dissociation and umbrella modes, was constructed. For every $R$ distance, the vibrational levels in the umbrella mode were estimated using the Fourier Grid Hamiltonian method [54] in the $r$ grid. 


\section{RESULTS}

\section{A. Experimental results}

The experimental observables are the $\mathrm{CH}_{3}$ fragment VMI images obtained after photodissociation of $\mathrm{CH}_{3} \mathrm{I}$ excited at the center $(268 \mathrm{~nm})$ and at the blue side $(243 \mathrm{~nm})$ of the $A$-band, followed by vibrationally-state resolved resonant multiphoton ionization of the $\mathrm{CH}_{3}(\nu)$ fragment in a pump-probe fashion as a function of the time delay. Since the results at $268 \mathrm{~nm}$ have been the subject of previous publications [14-16], more emphasis will be placed here on the results at $243 \mathrm{~nm}$ and the comparison with respect to the center-of-the-band excitation. The first part of this section will present results concerning the formation of vibrationless methyl fragments $(\nu=0)$, and the second part will describe the formation of umbrella mode excited methyl $\left(\nu_{2}\right)$.

The inverted $\mathrm{CH}_{3}(\nu=0)$ fragment images obtained from the photodissociation of $\mathrm{CH}_{3} \mathrm{I}$ at $243 \mathrm{~nm}$ and $268 \mathrm{~nm}$ detected via $(2+1)$ REMPI at $333.5 \mathrm{~nm}$ ( $Q$ branch of the twophoton $3 p_{z}{ }^{2} A_{2}^{\prime \prime} \leftarrow^{2} A_{2}$ " $0_{0}^{0}$ transition) for an asymptotic pump-probe time delay, i.e. when the reaction can be regarded as finished and the fragments enough separated at a distance implying a negligible interaction between them (resonant condition in Femtochemistry for the clocking of the reaction), are shown in Figure 2. The weak signal in the center of the images is attributed to dissociative ionization processes, which are not the subject of the present study, and thus in the following we shall concentrate on the higher kinetic energy rings. The corresponding center-of-mass methyl fragment translational energy distributions (TED) resulting from the angular integration of the inverted images are depicted in the right panels of Figure 2. Maximum available energies expected for each channel were obtained from literature values for dissociation energy, iodine spin-orbit splitting and $\mathrm{CH}_{3}$ vibrational quanta $[10,15]$, and upon neglecting the internal energy content of the parent molecule. They are indicated with dashed vertical lines in Figure 2.

Nanosecond experiments with $(2+1)$ REMPI $\mathrm{CH}_{3}(\nu=0)$ detection report two channels as shown by the more intense inner and outer rings, which are assigned to the channels $\mathrm{CH}_{3}(\nu=0)+\mathrm{I}^{*}\left({ }^{2} P_{1 / 2}\right)$ and $\mathrm{CH}_{3}(\nu=0)+\mathrm{I}\left({ }^{2} P_{3 / 2}\right)$ (indicated, respectively, by (3) and (1) in Figures $2 \mathrm{~b}$ and $2 \mathrm{~d})[10,11]$. However, for femtosecond pulse probing, it has already been shown that a third middle ring is distinguished after excitation at $268 \mathrm{~nm}$ and assigned to the chan- 

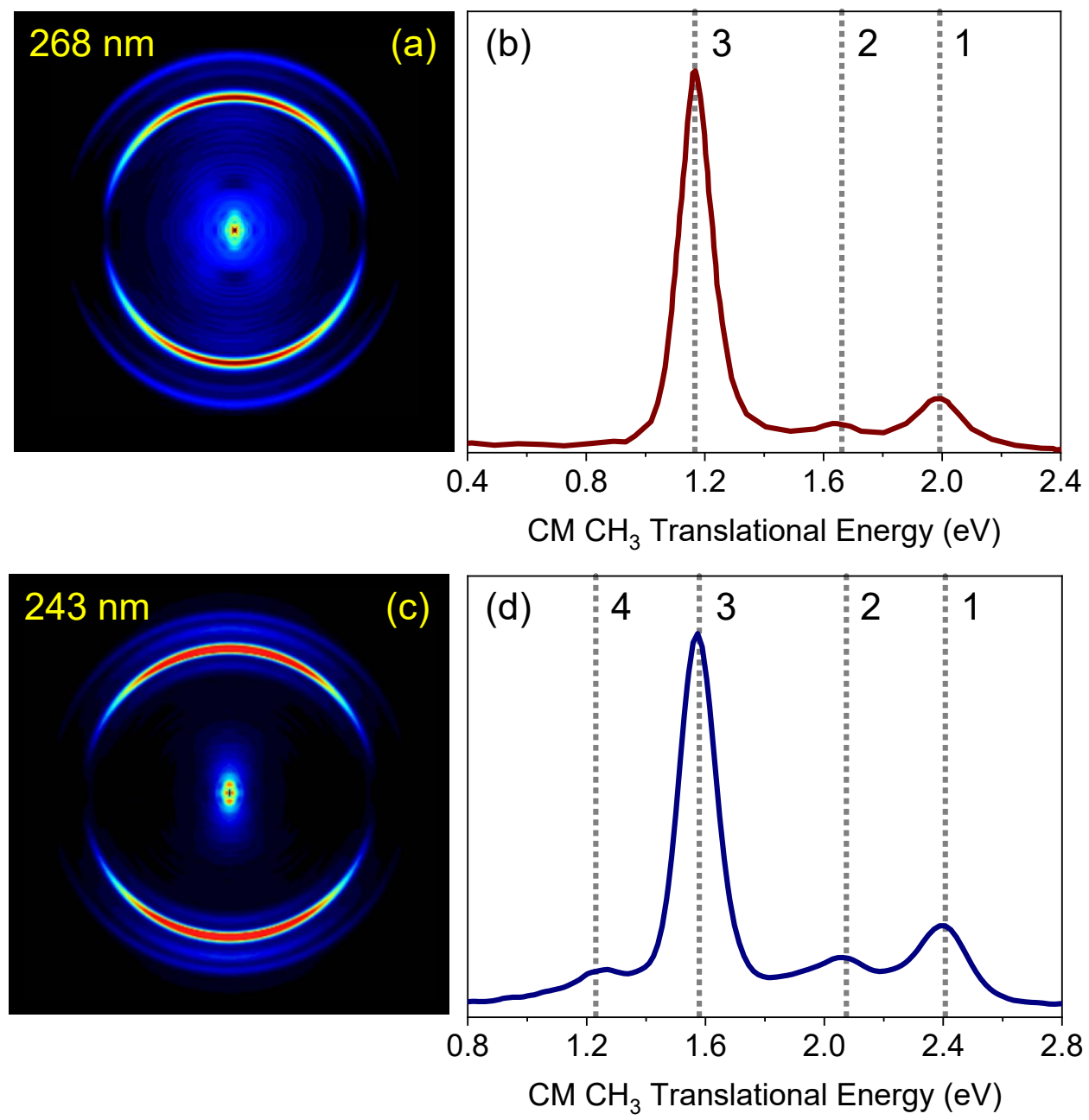

FIG. 2: Asymptotic Abel inverted $\mathrm{CH}_{3}$ image and corresponding center-of-mass translational energy distribution (TED) obtained upon $\mathrm{CH}_{3} \mathrm{I}$ excitation at (a,c) $268 \mathrm{~nm}$ and (b,d) 243 $\mathrm{nm}$, and $\mathrm{CH}_{3}(2+1)$ REMPI detection at $333.5 \mathrm{~nm}$ and at 10 ps pump-probe time delay. The polarization axis of the pump and probe pulses is vertical. Contributions to the images and TEDs are indicated by dashed vertical lines and numbered according to the following assignments: (1) $\mathrm{CH}_{3}(\nu=0)+\mathrm{I}\left({ }^{2} P_{3 / 2}\right) ;(2) \mathrm{CH}_{3}\left(\nu_{1}=1\right)+\mathrm{I}\left({ }^{2} P_{3 / 2}\right) ;(3) \mathrm{CH}_{3}(\nu=0)+\mathrm{I}^{*}\left({ }^{2} P_{1 / 2}\right)$; (4) $\mathrm{CH}_{3}\left(\nu_{1}=1\right)+\mathrm{I}^{*}\left({ }^{2} P_{1 / 2}\right)$. Detection of $\mathrm{CH}_{3}\left(\nu_{1}=1\right)$ with a $(2+1)$ REMPI for the $1_{1}^{1}$ band at $333.9 \mathrm{~nm}$, is accidental due to the bandwidth of the probe femtosecond laser pulse centered at $333.5 \mathrm{~nm}$. 
nel yielding $\mathrm{CH}_{3}\left(\nu_{1}=1\right)+\mathrm{I}\left({ }^{2} P_{3 / 2}\right)[14,15]$ (indicated as $(2)$ in Figures $2 \mathrm{~b}$ and $2 \mathrm{~d}$ ). This ring is detectable accidentally due to the spectral proximity of the $1_{1}^{1}$ and $0_{0}^{0}$ bands of $\mathrm{CH}_{3}$, both within the bandwidth of the femtosecond probe laser [14, 15]. Upon excitation at $243 \mathrm{~nm}$, a fourth weak ring with the lowest translational energy can be observed and attributed to the channel yielding symmetric-stretch mode excited $\mathrm{CH}_{3}\left(\nu_{1}=1\right)$ in correlation with $\mathrm{I}^{*}\left({ }^{2} P_{1 / 2}\right)$. This new photodissociation channel, marked as (4) in Figure 2d, is open due to the larger amount of energy available for the fragments with this shorter excitation wavelength.

Analogous images were measured for the probe laser tuned to the $Q$ branch of the twophoton $3 p_{z}{ }^{2} A_{2} " \leftarrow^{2} A_{2} " 2_{1}^{1}$ and $2_{2}^{2}$ transitions for $\mathrm{CH}_{3}$, i.e. predominantly $(2+1)$ REMPI probing umbrella mode-excited $\mathrm{CH}_{3}\left(\nu_{2}=1\right)$ and $\mathrm{CH}_{3}\left(\nu_{2}=2\right)$, at wavelengths 329.4 and 325.8 $\mathrm{nm}$, respectively [15]. These images are not shown for brevity, but they are very similar to those measured for excitation at $268 \mathrm{~nm}$ excitation reported in our previous work [15]. In the present case, for excitation at $243 \mathrm{~nm}$, there appear rings associated to the channels $\mathrm{CH}_{3}\left(\nu_{2}=1,2\right)+\mathrm{I}\left({ }^{2} P_{3 / 2}\right), \mathrm{CH}_{3}\left(\nu_{1}=1, \nu_{2}=1,2\right)+\mathrm{I}\left({ }^{2} P_{3 / 2}\right), \mathrm{CH}_{3}\left(\nu_{2}=1,2\right)+\mathrm{I}^{*}\left({ }^{2} P_{1 / 2}\right)$ and $\mathrm{CH}_{3}\left(\nu_{1}=1, \nu_{2}=1,2\right)+\mathrm{I}^{*}\left({ }^{2} P_{1 / 2}\right)$.

As is clear from the images depicted in Figure 2, the angular character of all the $A$-band photodissociation contributions observed is parallel (i.e. maximizing along the polarization axis of the electric field of the laser) for excitation at $268 \mathrm{~nm}$ and at $243 \mathrm{~nm}$. The angular distributions can be obtained by radial integration of the images across each of the selected ranges for each channel. A detailed analysis of the angular distributions of the different dissociation channels has been carried out considering the possibility of polarization effects for the $\mathrm{CH}_{3}$ fragment ascribed to rotational alignment $[12,13]$. The angular distributions have been fitted to the commonly used expression for one-photon dissociation and a $(2+1)$ REMPI detection process $[12,13]$ :

$$
I(\theta)=\frac{\sigma}{4 \pi}\left[1+\beta_{2} P_{2}(\cos \theta)+\beta_{4} P_{4}(\cos \theta)+\beta_{6} P_{6}(\cos \theta)\right]
$$

where $\theta$ is the angle between the photofragment recoil direction and the photolysis polarization direction, $\sigma$ is the absorption cross section (since the experimental setup has not been calibrated for total intensities, the quotient has been treated as a normalization fitting parameter), $\beta_{i}$ are anisotropy parameters which reflect the dissociation dynamics and the photofragment polarization, and $P_{i}$ are the Legendre polynomials of $i$ th order. If no photofragment polarization is expected, Eq. (1) can be truncated in $i=2$, and for this 
particular case, $\beta_{2}$ coincides with the anisotropy parameter $\beta$.

The anisotropy parameter values obtained from the fits of the angular distributions for the dissociation channels yielding $\mathrm{CH}_{3}(\nu=0)$ and $\mathrm{CH}_{3}\left(\nu_{2}=1\right)$ for the excitation wavelengths 243 and $268 \mathrm{~nm}$ are depicted in Figure 3 along with those obtained in nanosecond experiments in the red and blue edges of the $A$-band $[12,13]$. As can be seen, the $\beta_{4}$ and $\beta_{6}$ values returned from the fit at the two excitation wavelengths studied in this work (243 and $268 \mathrm{~nm}$ ) are small and approximately constant, which indicates that fragment polarization effects are minor. Therefore, the photodissociation anisotropy parameter $\beta$ should be closely connected to the measured $\beta_{2}$ parameter. The large values of $\beta_{2}$, being indeed around the limiting value of 2 allowed for a pure parallel transition, for both the $I^{*}\left({ }^{2} P_{1 / 2}\right)$ and $I\left({ }^{2} P_{3 / 2}\right)$ channels and for both $\mathrm{CH}_{3}$ in $\nu=0$ and $\nu_{2}=1$ vibrational states, clearly state that excitation is carried out predominately to the ${ }^{3} Q_{0}$ state and consequently no participation of direct dissociation from the perpendicular ${ }^{1} Q_{1}$ state is expected at the two excitation wavelengths studied in this work. The decrease of the $\beta_{2}$ values measured in the red and blue edges of the $A$-band $[12,13]$ depicted in Figure 3 highlight indeed the increasing participation of the perpendicular ${ }^{3} Q_{1}$ and ${ }^{1} Q_{1}$ states, respectively, when reaching the limits of excitation at $333.45 \mathrm{~nm}$ and 217 $\mathrm{nm}$, respectively.

Methyl images detected at $333.5 \mathrm{~nm}$ have been recorded as a function of the pumpprobe time delay for both excitation to the center and the blue side of the absorption band. The results are presented in Figure 4 for the case of excitation at $243 \mathrm{~nm}$. It is observed that at very short time delays, the dominant contribution in the images is multiphoton dissociative ionization, which appears in the center of the image, but as time delay increases, this contribution gradually decreases and eventually disappears and at the same time the intensity of the rings becomes stronger. Similar sets of time-dependent images were acquired for the probe laser wavelengths 329.4 and $325.8 \mathrm{~nm}$, respectively, for detection of $\mathrm{CH}_{3}\left(\nu_{2}=1\right)$ and $\mathrm{CH}_{3}\left(\nu_{2}=2\right)$ (not shown).

The images were angularly integrated to obtain the corresponding $\mathrm{CH}_{3}$ translational energy distributions (TEDs) for each time delay. Figure 5 shows the time-dependent TEDs in the form of 2D maps for all three probe wavelengths (333.5, 329.5 and $325.8 \mathrm{~nm}$ ) for excitation at $243 \mathrm{~nm}$.

For the analysis of the maps shown in Figure 5, a multidimensional fitting method has been employed through parameterized functions that describe each of the contributions to 


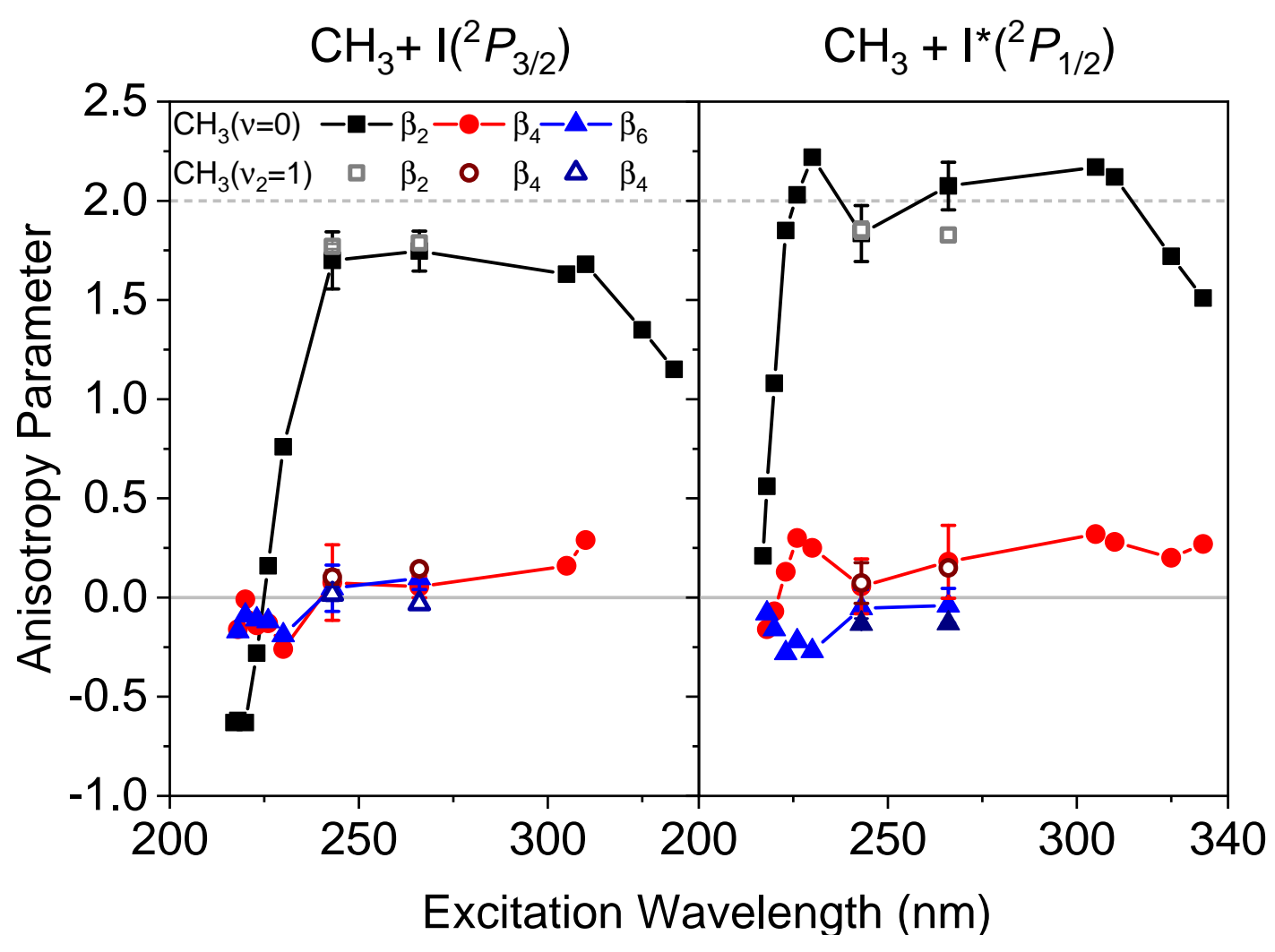

FIG. 3: Anisotropy parameters $\beta_{2}, \beta_{4}$ and $\beta_{6}$ obtained from the fit of the angular distributions extracted from the asymptotic images measured at 333.5 and $329.5 \mathrm{~nm}$ probing, i.e. for detection of $\mathrm{CH}_{3}(\nu=0)$ (depicted in Figure 2) and $\mathrm{CH}_{3}\left(\nu_{2}=1\right.$ ) (not shown). Error bars represent the standard deviation of the analysis of several measurements. The present anisotropy values obtained at excitation wavelengths 243 and $268 \mathrm{~nm}$ are represented together with those reported in Refs. $[12,13]$ from nanosecond experiments in the red and blue edges of the absorption $A$-band.
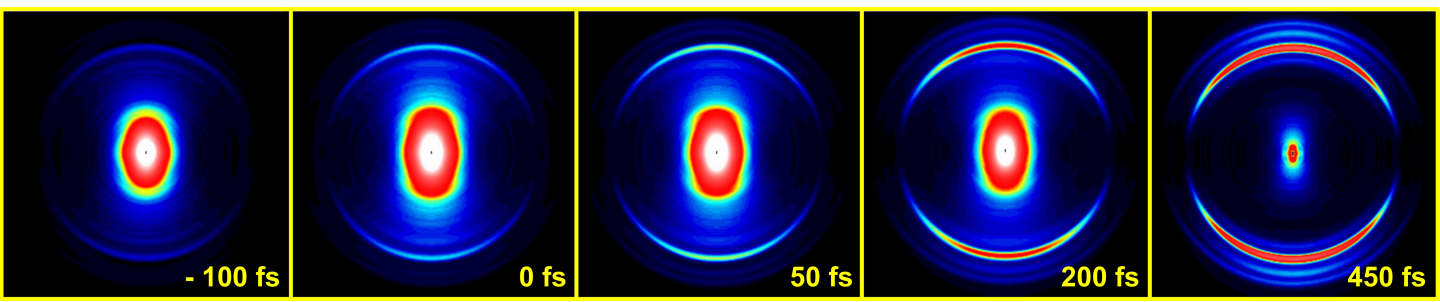

FIG. 4: Sequence of Abel-inverted $\mathrm{CH}_{3}$ images obtained upon $\mathrm{CH}_{3} \mathrm{I}$ excitation at $243 \mathrm{~nm}$ and $\mathrm{CH}_{3}$ detection by $(2+1)$ REMPI at $333.5 \mathrm{~nm}$ as a function of the indicated pump-probe time delay. 

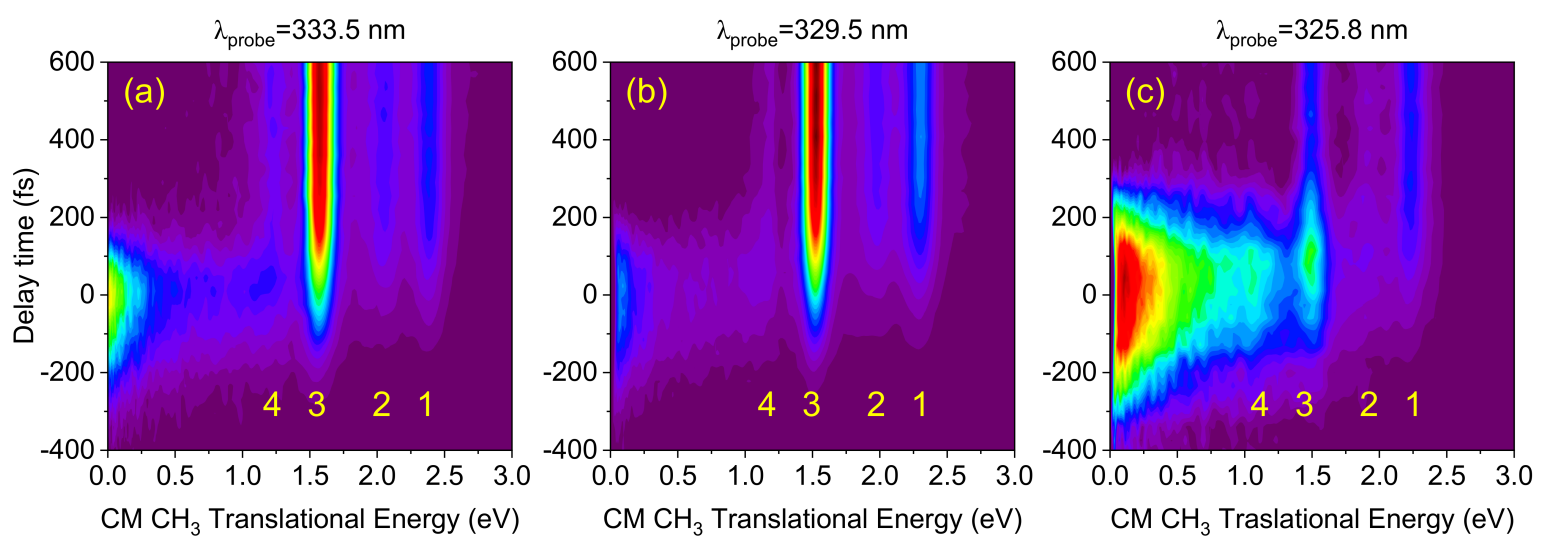

FIG. 5: 2D maps of the center-of-mass (CM) translational energy distributions of $\mathrm{CH}_{3}$ upon excitation of $\mathrm{CH}_{3} \mathrm{I}$ at $243 \mathrm{~nm}$ as a function of time delay for $(2+1)$ REMPI probing of methyl at (a) $333.5 \mathrm{~nm}$, (b) $329.5 \mathrm{~nm}$ and (c) $325.8 \mathrm{~nm}$. The vertical peaks shown in (a) correspond to the four rings depicted in Figures 2c and 4. Vibrationless $\nu=0$ methyl is visible, formed in correlation with ground $\mathrm{I}\left({ }^{2} P_{3 / 2}\right)$ and spin-orbit excited $\mathrm{I}^{*}\left({ }^{2} P_{1 / 2}\right)$ fragments (labelled 1 and 3 , respectively). Methyl with one quantum in the symmetric stretch mode $\nu_{1}=1$ is formed in correlation with ground $\mathrm{I}\left({ }^{2} P_{3 / 2}\right)$ and spin-orbit excited $\mathrm{I}^{*}\left({ }^{2} P_{1 / 2}\right)$ (labelled 2 and 4 , respectively). In (b) the observed channels are $\mathrm{CH}_{3}\left(\nu_{2}=1\right)+\mathrm{I}\left({ }^{2} P_{3 / 2}\right) / \mathrm{I}^{*}\left({ }^{2} P_{1 / 2}\right)$ (labelled 1 and 3$)$ and $\mathrm{CH}_{3}\left(\nu_{1}=1, \nu_{2}=1\right)+\mathrm{I}\left({ }^{2} P_{3 / 2}\right) / \mathrm{I}^{*}\left({ }^{2} P_{1 / 2}\right)$ (labelled 2 and 4 ) and in (c) the observed channels are $\mathrm{CH}_{3}\left(\nu_{2}=2\right)+\mathrm{I}\left({ }^{2} P_{3 / 2}\right) / \mathrm{I}^{*}\left({ }^{2} P_{1 / 2}\right)$ (labelled 1 and 3) and $\mathrm{CH}_{3}\left(\nu_{1}=1, \nu_{2}=2\right)+\mathrm{I}\left({ }^{2} P_{3 / 2}\right)$ (labelled 2).

the image radially, angularly and temporally. The minimization algorithm employed to find optimum parameters is a Levenberg-Marquardt nonlinear regression method that is applied globally to the complete set of images. The method has been described previously in detail [36]. In this work, it has proven essential to deal with the full Abel-inverted images, and not only with the TEDs, due to overlapping contributions that could only be disentangled through the consideration of the angular coordinate [55]. The main values of interest to extract from such analysis are the appearance (clocking) times of each of the channels. For this matter, resonant contributions were temporally described with a Boltzmann sigmoidal curve of the form

$$
S=A_{2}+\frac{A_{1}-A_{2}}{1+e^{\left(t-t_{0}\right) / t_{c}}}
$$

which is characterized by a center temporal position $t_{0}$, i.e. the time delay for which the 
intensity has reached half its asymptotic value, and a rise time constant $t_{c}$. Their radial behavior was modeled with Gaussian functions, and angularly they were described as a combination of even Legendre polynomials up to fourth order.

The transients obtained for $243 \mathrm{~nm}$ excitation using this method are shown in Figure 6, where the different curves correspond to the channels observed and listed in Table I. The solid lines correspond to the temporal amplitude of each component as fitted with optimized parameters according to Eq. (2). The dashed vertical lines mark the optimized value of $t_{0}$ in each case. The points on the graph are obtained as the result of integrating the global intensity of the experimental image at each delay and subtracting the sum of all other components as fitted through the global fit procedure described above. Simulation of the central part of the image, where the signal originates from multiphoton dissociative ionization processes, is shown as the first curve on the top of each panel, and its maximum is take as the reference time or time zero. As the transients in Figure 6 show, all measured appearance times are in the 50-145 fs range, the shortest clocking times corresponding to the measurements performed at the probe wavelength of $333.5 \mathrm{~nm}$ for detection of vibrationless $\mathrm{CH}_{3}$.

Similar measurements and analysis were performed for excitation at $268 \mathrm{~nm}$, revisiting those carried out in the past in our group [15], but with a better cross correlation between the pump and probe pulses, better signal-to-noise ratios, and a careful analysis of the data following the procedure indicated above based on the multidimensional fitting strategy reported in Ref. [36].

The main quantitative results obtained from the data acquired at the center of the absorption band (268 $\mathrm{nm}$ excitation) and the blue side of the absorption band (243 $\mathrm{nm}$ excitation) are summarized in Table I. In the table, the experimental appearance (clocking) times, $\tau$, for all quantum (vibrational and electronic) state-resolved channels at both excitation wavelengths, the differences between clocking times, $\Delta \tau$, and the I/I* ratios are presented. Figure 7 depicts the clocking times of the different state-resolved channels as a function of the vibrational energy content of the $\mathrm{CH}_{3}$ fragment.

As can be seen in Table I and Figure 7, the appearance times measured for the formation of vibrationless $\mathrm{CH}_{3}(\nu=0)$ follow the expected trend as a function of the increasing excitation energy, i.e. a reduction of the reaction time as more energy is available to be transformed into translational energy. This is valid for the comparison between ground state $\mathrm{I}\left({ }^{2} P_{3 / 2}\right)$ 

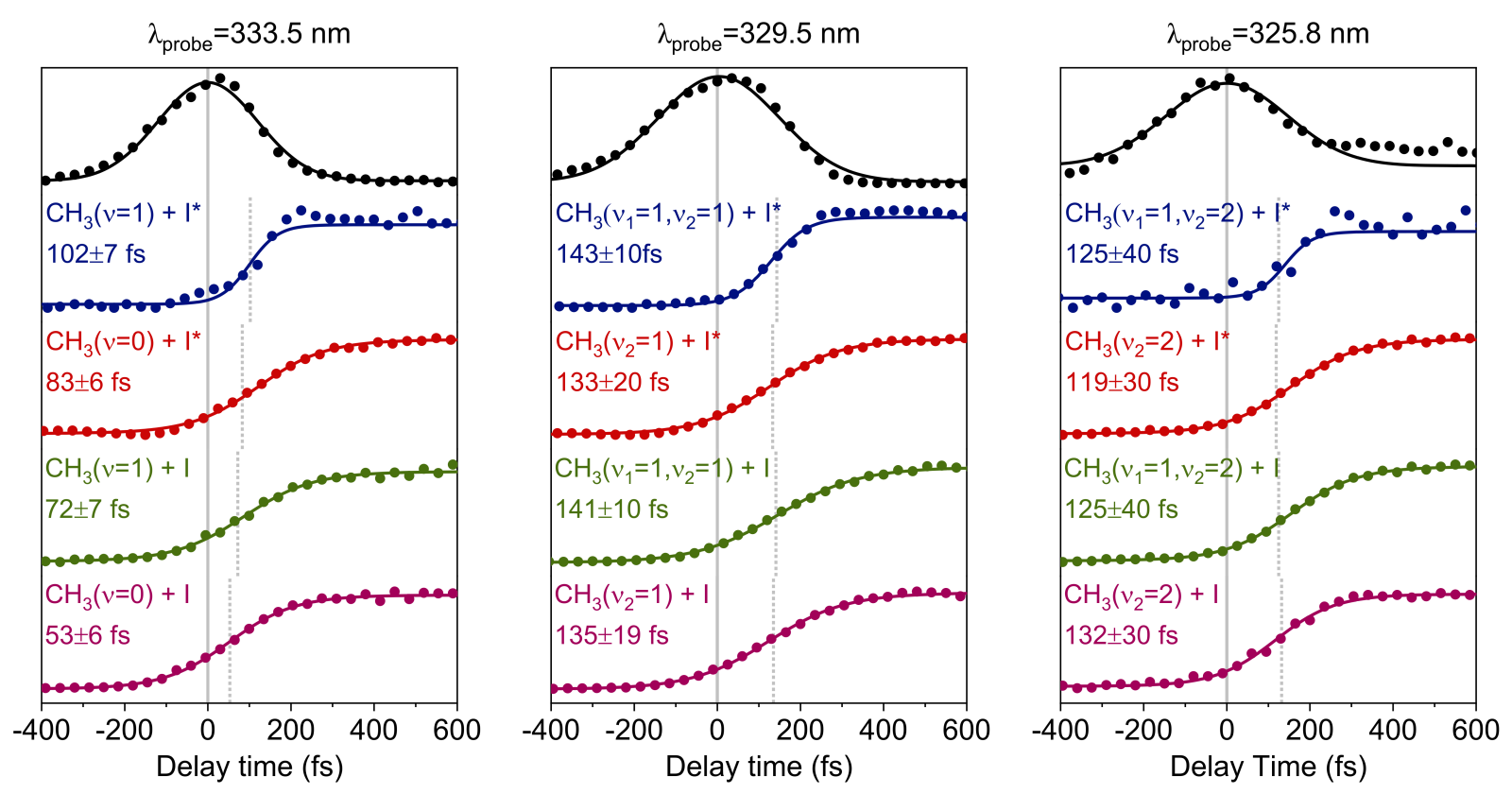

FIG. 6: $\mathrm{CH}_{3}$ transients corresponding to the dissociation channels indicated observed upon (2+1) REMPI probing at $333.5 \mathrm{~nm}, 329.5 \mathrm{~nm}$ and $325.8 \mathrm{~nm}$ after excitation of $\mathrm{CH}_{3} \mathrm{I}$ at $243 \mathrm{~nm}$. The multiphoton dissociative ionization transients measured set the time zero for our clock, which is indicated by the solid vertical line in each plot. The dashed vertical lines in each transient represent the half of the rise of the sigmoidal signal and therefore give the appearance times with respect to time zero. The clocking times (with statistical uncertainties) are indicated in each transient.

and spin-orbit excited state $I^{*}\left({ }^{2} P_{1 / 2}\right)$ at a given excitation energy, with systematically longer reaction times for $\mathrm{I}^{*}\left({ }^{2} P_{1 / 2}\right)$ - about $30-35 \mathrm{fs}$ - and is also valid for the comparison between the center $(268 \mathrm{~nm})$ and the blue side $(243 \mathrm{~nm})$ absorption of the $A$-band, with shorter times as more available energy is introduced into the system, i.e. faster dissociation after absorption in the blue side of the band.

The interpretation of the reaction times measured for vibrationally excited methyl channels (in $\nu_{1}$ and $\nu_{2}$ modes) is considerably more complex. The best example is the relative appearance times $(\Delta \tau)$ measured for $\mathrm{CH}_{3}\left(\nu_{2}>0\right)$ in correlation with ground state $\mathrm{I}\left({ }^{2} P_{3 / 2}\right)$ and spin-orbit excited $I^{*}\left({ }^{2} P_{1 / 2}\right)$, which show a decreasing trend as vibrational excitation in $\nu_{2}$ increases, being practically 0 fs for $\mathrm{CH}_{3}\left(\nu_{2}=1\right)$ and even inverting their appearance times for $\mathrm{CH}_{3}\left(\nu_{2}=2\right)$, at both $268 \mathrm{~nm}$ and $243 \mathrm{~nm}$ excitation. Interestingly, the same trend in the relative appearance times occur for $\mathrm{CH}_{3}\left(\nu_{1}=1\right)$ as excitation in $\nu_{2}$ increases, as can be 
TABLE I: Appearance (clocking) times $\tau$ (in fs), differences between appearance times $\Delta \tau$ and I/I* branching ratios measured for the different channels of the $A$-band photodisociation of $\mathrm{CH}_{3} \mathrm{I}$ at the excitation wavelengths $\lambda_{\text {exc }} 268 \mathrm{~nm}$ and $243 \mathrm{~nm}$. Statistical uncertainties obtained from various experiments carried out in each case are indicated.

\begin{tabular}{|c|c|c|c|c|c|c|c|}
\hline \multicolumn{8}{|c|}{$\lambda_{e x c}=268 \mathrm{~nm}$} \\
\hline & $\mathrm{CH}_{3}\left(\nu_{1}, \nu_{2}\right)$ & $(0,0)$ & $(1,0)$ & $(0,1)$ & $(1,1)$ & $(0,2)$ & $(1,2)$ \\
\hline $\mathrm{I}\left({ }^{2} P_{3 / 2}\right), \tau(\mathrm{fs})$ & & $75 \pm 19$ & $127 \pm 21$ & $84 \pm 12$ & $182 \pm 10$ & $102 \pm 9$ & $202 \pm 3$ \\
\hline $\mathrm{I}^{*}\left({ }^{2} P_{1 / 2}\right), \tau(\mathrm{fs})$ & & $114 \pm 19$ & - & $84 \pm 17$ & - & $93 \pm 12$ & - \\
\hline$\Delta \tau(\mathrm{fs})$ & & $34 \pm 38$ & - & $0 \pm 29$ & - & $-9 \pm 21$ & - \\
\hline $\mathrm{I} / \mathrm{I}^{*}$ ratio & & 0.22 & - & 0.33 & - & 1.15 & - \\
\hline \multicolumn{8}{|c|}{$\lambda_{e x c}=243 \mathrm{~nm}$} \\
\hline & $\mathrm{CH}_{3}\left(\nu_{1}, \nu_{2}\right)$ & $(0,0)$ & $(1,0)$ & $(0,1)$ & $(1,1)$ & $(0,2)$ & $(1,2)$ \\
\hline $\mathrm{I}\left({ }^{2} P_{3 / 2}\right), \tau(\mathrm{fs})$ & & $53 \pm 6$ & $72 \pm 7$ & $135 \pm 19$ & $141 \pm 10$ & $132 \pm 30$ & $125 \pm 40$ \\
\hline $\mathrm{I}^{*}\left({ }^{2} P_{1 / 2}\right), \tau(\mathrm{fs})$ & & $83 \pm 6$ & $102 \pm 7$ & $133 \pm 20$ & $143 \pm 10$ & $119 \pm 30$ & $125 \pm 40$ \\
\hline$\Delta \tau(\mathrm{fs})$ & & $30 \pm 12$ & $30 \pm 14$ & $-2 \pm 39$ & $2 \pm 20$ & $-13 \pm 7$ & $0 \pm 80$ \\
\hline $\mathrm{I} / \mathrm{I}^{*}$ ratio & & 0.22 & & 0.39 & & 1.07 & \\
\hline
\end{tabular}

inspected in the right part of Figure 7 and in Table I.

We would like to highlight an important difference between the appearance times measured at 243 and $268 \mathrm{~nm}$. Whereas $\tau$ decreases as $\nu_{2}$ excitation increases for $\nu_{1}=0$ at $268 \mathrm{~nm}$ excitation, in agreement with previous observations [15], the opposite trend is observed for both $\nu_{1}=0$ and $\nu_{1}=1$ at $243 \mathrm{~nm}$ excitation. Indeed, $\tau$ increases substantially when passing from $\mathrm{CH}_{3}(\nu=0)$ to $\mathrm{CH}_{3}\left(\nu_{2}=1\right)$ for both $\left.\mathrm{I}^{2} P_{3 / 2}\right)$ and $\mathrm{I}^{*}\left({ }^{2} P_{1 / 2}\right)$ channels. Considering the decrease in $\tau$ observed at $243 \mathrm{~nm}$ for vibrationless $\mathrm{CH}_{3}(\nu=0)$ in comparison with excitation at $268 \mathrm{~nm}$, commented on above, umbrella-mode vibrational excitation in $\mathrm{CH}_{3}$ implies a quite substantial and unexpected increase in the clocking time at this excitation wavelength, which is absent at $268 \mathrm{~nm}$ excitation. Specifically, clocking times go from $53 \pm 6$ fs to $135 \pm 19$ fs and from $83 \pm 6$ fs to $133 \pm 20$ fs for the $\mathrm{I}\left({ }^{2} P_{3 / 2}\right)$ and $\mathrm{I}^{*}\left({ }^{2} P_{1 / 2}\right)$ channels, respectively, when the methyl umbrella mode is excited with one quantum.

It is interesting to note that these apparent anomalies in the clocking times appear 


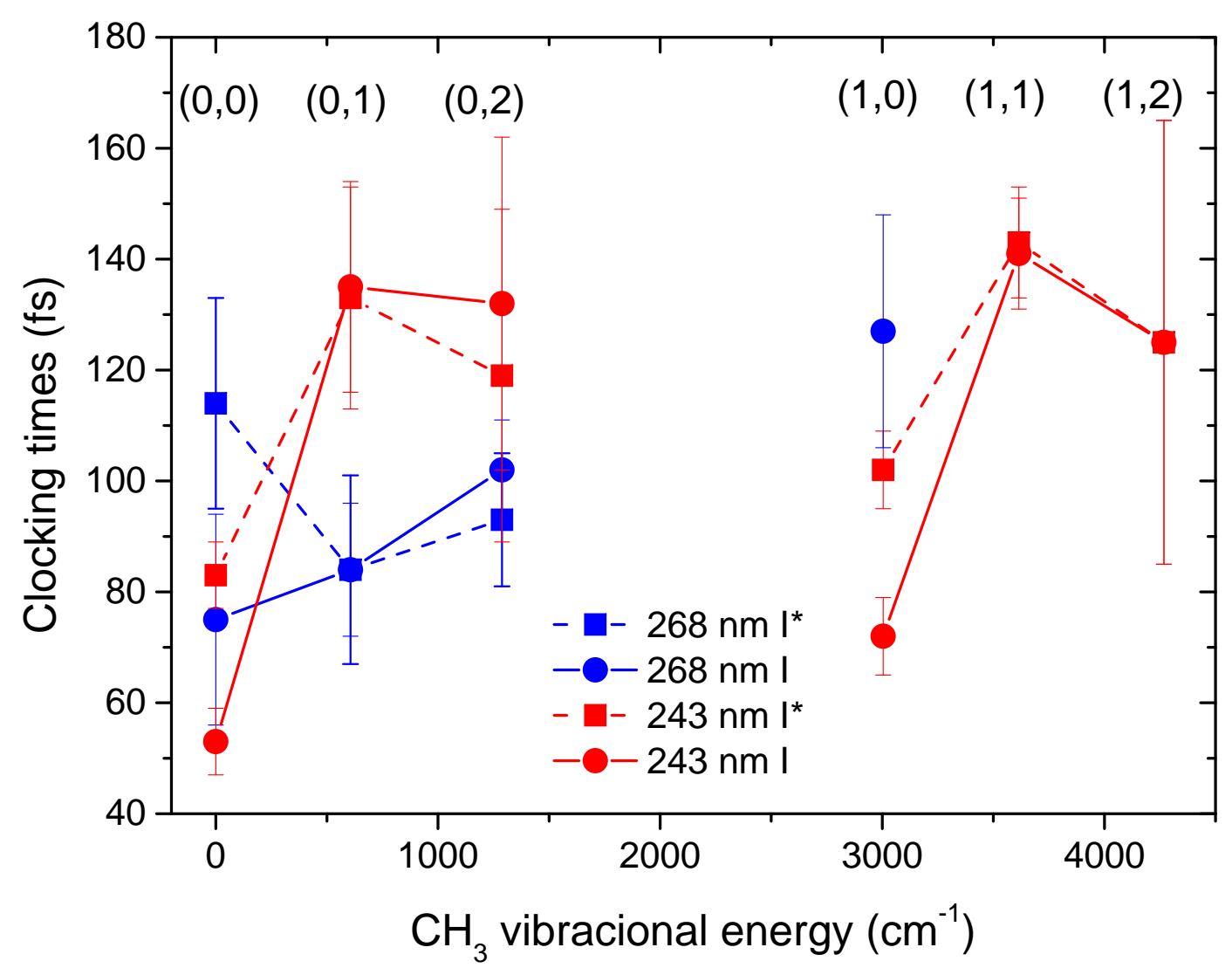

FIG. 7: Appearance (clocking) times (with statistical error bars) measured for the different $\mathrm{CH}_{3}\left(\nu_{1}, \nu_{2}\right)+\mathrm{I} / \mathrm{I}^{*}$ channels of the photodissociation of $\mathrm{CH}_{3} \mathrm{I}$ at $268 \mathrm{~nm}$ and $243 \mathrm{~nm}$ as a function of the excess vibrational energy of the $\mathrm{CH}_{3}$ fragment.

when observation of the process is made through the umbrella mode excited components of the outgoing vibrational wavepacket. Indeed, it is this mode that shows the highest activity as dissociation proceeds, reflecting the fact that the $\mathrm{CH}_{3}$ fragment undergoes a dramatic change in its equilibrium geometry, from pyramidal to planar. Thus, as absorption to the $\mathrm{CH}_{3} \mathrm{I} A$-band takes place, a hot vibrational wavepacket is launched, with most of the excitation concentrated in the umbrella mode. This mode is characterized by a relatively low vibrational frequency and a correspondingly long vibration period ( $55 \mathrm{fs}$ ), which is of the order of the dissociation times. Moreover, a vibrational inversion is known to occur after the non-adiabatic transition from the ${ }^{3} Q_{0}$ to the ${ }^{1} Q_{1}$ states, which characterizes the appearance of ground state $\mathrm{I}\left({ }^{2} P_{3 / 2}\right)$ fragments. 


\section{B. Theoretical results}

\section{1. $4 D$ wavepacket calculations}

The theoretical appearance times, $\tau$, for the $\mathrm{I}\left({ }^{2} P_{3 / 2}\right)$ and $\mathrm{I}^{*}\left({ }^{2} P_{1 / 2}\right)$ dissociation channels, the differences between appearance times, $\Delta \tau$, and the I/I* branching ratios obtained with the $4 \mathrm{D}$ wavepacket method are collected in Table II and depicted in the top panel of Figure 8 for the excitation wavelengths $243 \mathrm{~nm}$ and $268 \mathrm{~nm}$.

For both wavelengths, the times associated with the $\mathrm{I}\left({ }^{2} P_{3 / 2}\right)$ and $\mathrm{I}^{*}\left({ }^{2} P_{1 / 2}\right)$ channels increase monotonically and smoothly with increasing vibrational excitation of the methyl fragment. The longer appearance times obtained for the reaction yielding vibrationally excited $\nu_{1}=1$ methyl agree with the experimental results. However, the monotonic increase of the wave packet appearance times $\tau$ when $\nu_{2}$ excitation in methyl increases is in contradiction with the non-monotonic behavior observed experimentally (see Figure 7), as it was also found previously $[15,16]$.

TABLE II: 4D wavepacket calculated appearance times $\tau$ (in fs), differences between appearance times $\Delta \tau$ and $\mathrm{I} / \mathrm{I}^{*}$ branching ratios measured for the different channels of the $A$-band photodisociation of $\mathrm{CH}_{3} \mathrm{I}$ at the excitation wavelengths $\lambda_{e x c}=268 \mathrm{~nm}$ and $243 \mathrm{~nm}$.

\begin{tabular}{|c|c|c|c|c|c|}
\hline \multicolumn{6}{|c|}{$\lambda_{\text {exc }}=268 \mathrm{~nm}$} \\
\hline & $\mathrm{CH}_{3}\left(\nu_{1}, \nu_{2}\right)(0,0)$ & $(1,0)$ & $(0,1)(1,1)$ & $(0,2)$ & $(1,2)$ \\
\hline $\mathrm{I}\left({ }^{2} P_{3 / 2}\right), \tau(\mathrm{fs})$ & 94.2 & 103.2 & $95.4 \quad 104.7$ & 96.7 & 111.4 \\
\hline $\mathrm{I}^{*}\left({ }^{2} P_{1 / 2}\right), \tau(\mathrm{fs})$ & 114.3 & 129.4 & 117.1133 .8 & 121.8 & 142.2 \\
\hline$\Delta \tau(\mathrm{fs})$ & 20.1 & 26.2 & $21.7 \quad 29.1$ & 25.1 & 30.8 \\
\hline $\mathrm{I} / \mathrm{I}^{*}$ ratio & 0.06 & 0.03 & $2.01 \quad 1.41$ & 125.61 & 21.92 \\
\hline \multicolumn{6}{|c|}{$\lambda_{\text {exc }}=243 \mathrm{~nm}$} \\
\hline & $\mathrm{CH}_{3}\left(\nu_{1}, \nu_{2}\right)(0,0)$ & $(1,0)$ & $(0,1)(1,1)$ & $(0,2)$ & $(1,2)$ \\
\hline $\mathrm{I}\left({ }^{2} P_{3 / 2}\right), \tau(\mathrm{fs})$ & 86.1 & 93.9 & $\begin{array}{ll}86.9 & 93.8\end{array}$ & 87.8 & 95.6 \\
\hline $\mathrm{I}^{*}\left({ }^{2} P_{1 / 2}\right), \tau(\mathrm{fs})$ & 102.1 & 111.3 & 102.8114 .2 & 106.4 & 116.5 \\
\hline$\Delta \tau(\mathrm{fs})$ & 16.0 & 17.4 & $15.9 \quad 20.4$ & 18.6 & 20.9 \\
\hline $\mathrm{I} / \mathrm{I}^{*}$ ratio & 0.17 & 0.02 & $\begin{array}{ll}1.71 & 4.13\end{array}$ & 48.48 & 13.36 \\
\hline
\end{tabular}


The trend of the reaction times found theoretically is consistent with a monotonous and slight decrease of the energy available to the dissociation fragments as excitation in the vibrational modes of the $\mathrm{CH}_{3}$ fragment increases. Indeed, the appearance time increase is found to be rather small for excitation from $\nu_{2}=0$ to $\nu_{2}=2$ for the I and I* channels correlated with either $\mathrm{CH}_{3}\left(\nu_{1}=0\right)$ or $\mathrm{CH}_{3}\left(\nu_{1}=1\right)$, because these $\nu_{2}$ excitations involve little decrease of the energy available to the fragments. Excitation of the $\nu_{1}$ mode from $\nu_{1}=0$ to $\nu_{1}=1$ implies a somewhat larger decrease of the available energy, which is reflected in a more significant increase of the reaction times of Table II.

The longer appearance times obtained when the available energy decreases due to larger vibrational excitation of the $\mathrm{CH}_{3}$ fragment, is supported by the result found in Table II that the appearance time increases less with increasing vibrational excitation for $\lambda_{\text {exc }}=243 \mathrm{~nm}$ than for $\lambda_{e x c}=268 \mathrm{~nm}$. The reason is that the energy difference between vibrational levels is constant for the two wavelengths, and its contribution (in percentage) to the decrease of the total amount of available energy is smaller as the excitation wavelength becomes shorter (from 268 to $243 \mathrm{~nm}$ ), leading to a lower time increase.

The variation of the time difference $\Delta \tau$ between the I and $\mathrm{I}^{*}$ channels with vibrational excitation of the $\nu_{1}$ and $\nu_{2}$ modes follows the same trend for both excitation wavelengths. Similar arguments as the ones given above based on the amount of energy available to the fragments are applicable here. Indeed, similarly as with the appearance times $\tau$, variation of $\Delta \tau$ is also smaller for $\lambda_{\text {exc }}=243 \mathrm{~nm}$ than for $\lambda_{\text {exc }}=268 \mathrm{~nm}$. In addition, the $\Delta \tau$ values are somewhat lower in the case of $\lambda_{e x c}=243 \mathrm{~nm}$. Since the constant energy difference in the available energy to the fragments due to the spin-orbit splitting between the I and I* channels is what originates $\Delta \tau$, again its relative contribution to the total available energy is smaller for the shorter wavelength, and, thus, its effect on $\Delta \tau$ becomes correspondingly smaller.

The I/I* branching ratios also display the same behavior with vibrational excitation of the $\mathrm{CH}_{3}$ fragment for the two excitation wavelengths. The trend shown is that the ratio increases with increasing excitation in the umbrella mode, indicating that a higher vibrational excitation in that mode is produced through the I dissociation channel compared to the $\mathrm{I}^{*}$ one. The same trend is found in the experimental results of Table I, although the values of the theoretical ratios are in general quite different from the experimental ones. On the contrary, the theoretical I/I* ratio decreases in general upon excitation from $\nu=0$ to 

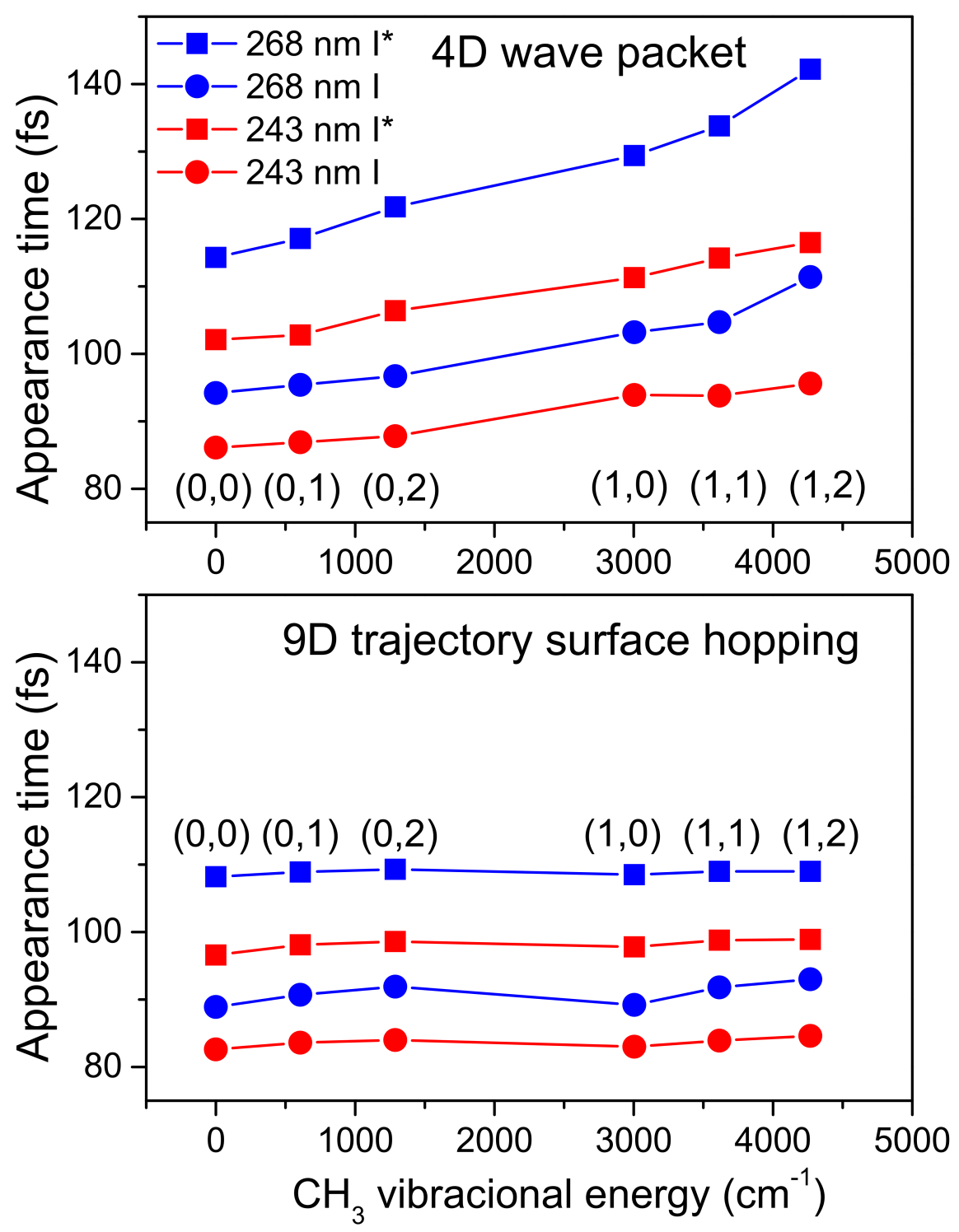

FIG. 8: Theoretical appearance times for the different $\mathrm{CH}_{3}\left(\nu_{1}, \nu_{2}\right)+\mathrm{I} / \mathrm{I}^{*}$ channels of the photodissociation of $\mathrm{CH}_{3} \mathrm{I}$ at $243 \mathrm{~nm}$ (red symbols) and $268 \mathrm{~nm}$ (blue symbols), as a function of the excess vibrational energy of the $\mathrm{CH}_{3}$ fragment. Top: 4D wavepacket calculations. Bottom: 9D surface hopping trajectory calculations. Values in parenthesis represent $\left(\nu_{1}, \nu_{2}\right)$ vibrational quantum numbers.

$\nu_{1}=1$ for the two excitation wavelengths.

Figure 9 displays the umbrella mode $\nu_{2}$ vibrational distributions for the channels with $\mathrm{CH}_{3}$ in $\nu_{1}=0$ and $\nu_{1}=1$ and I and I* at the two excitation wavelengths $243 \mathrm{~nm}$ and $268 \mathrm{~nm}$. As in previous works $[15,16,56,57]$, the vibrational distributions for the $I^{*}$ channels are 


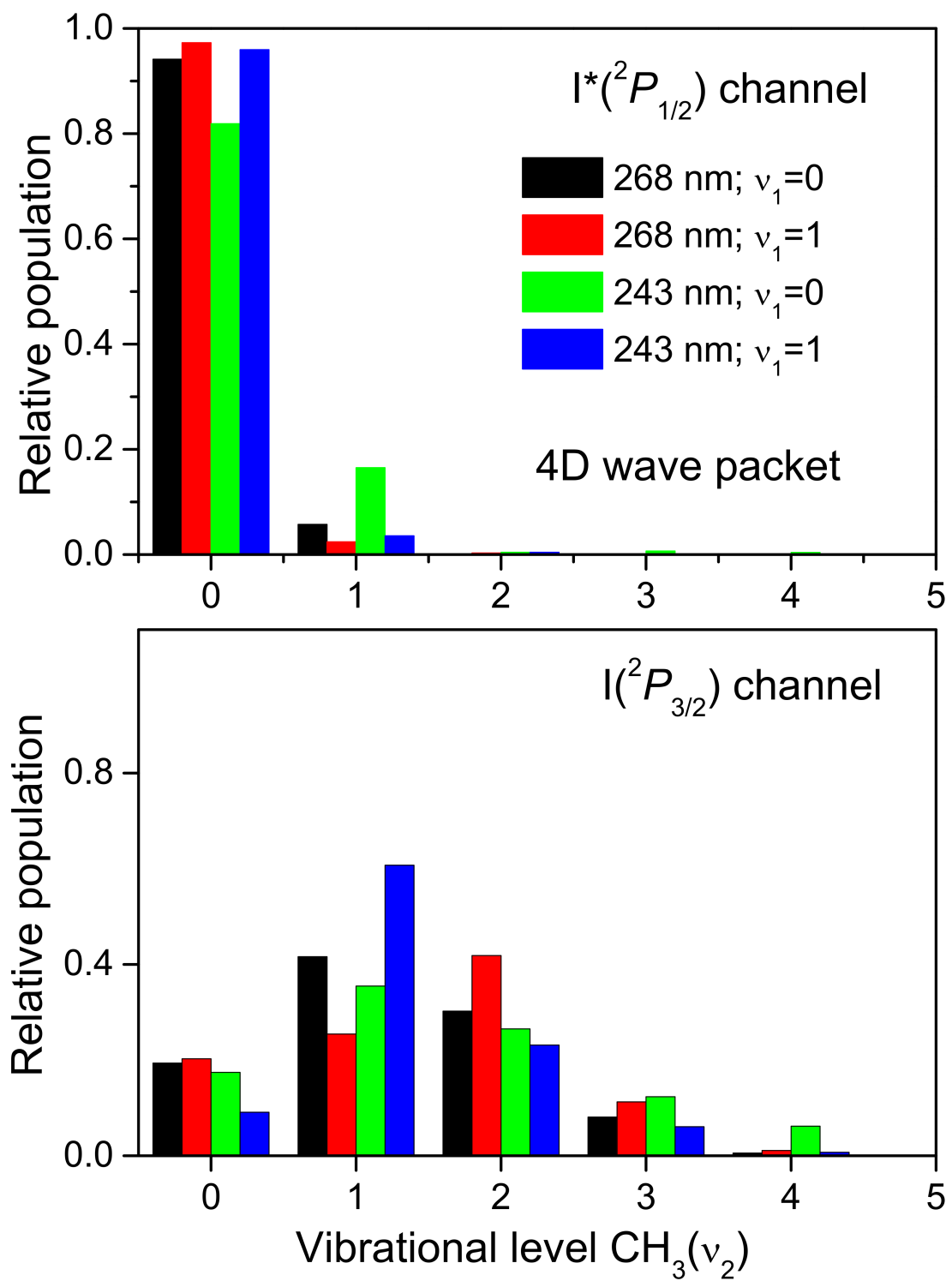

FIG. 9: Calculated vibrational distributions of the $\mathrm{CH}_{3}\left(\nu_{2}\right)$ fragments produced in the photodissociation of $\mathrm{CH}_{3} \mathrm{I}$ at the excitation wavelengths $268 \mathrm{~nm}$ and $243 \mathrm{~nm}$ through the $\mathrm{I}^{*}$ channel (upper panel) and the I channel (lower panel) using the $4 \mathrm{D}$ wave packet model.

rather cold peaking at $\nu_{2}=0$, irrespective the excitation wavelength. However, vibrational population inversion is observed for the I channels, the distributions peaking at $\nu_{2}=1$ or 2 . These observations are in general good agreement with the existing experimental data [57].

In summary, the present 4D wavepacket calculations carried out using the potential energy surfaces of Xie et al. [24] and the previously reported transition dipole moments [12], in which a long enough C-I distance has been considered for the calculation of the state-resolved 
(both vibrational and electronic) appearance times such that the interaction between the iodine and methyl fragments is negligible (13 bohr; $6.88 \AA$ ), demonstrate the minor role

of the ${ }^{3} Q_{0} /{ }^{1} Q_{1}$ conical intersection to influence the reaction times. Moreover, the small and monotonous trends observed in the theoretical appearance times as a function of vibrational excitation of the methyl fragment can be explained exclusively in terms of arguments based on the slightly different available energy in each case. Therefore, the observed nonmonotonous trend of the appearance times as a function of the vibrational energy content of the methyl fragment cannot be explained in terms of the passing through the conical intersection between the ${ }^{3} Q_{0}$ and ${ }^{1} Q_{1}$ states.

\section{2. $9 D$ surface-hopping trajectory calculations}

The theoretical appearance times, $\tau$, for the $\mathrm{I}\left({ }^{2} P_{3 / 2}\right)$ and $\mathrm{I}^{*}\left({ }^{2} P_{1 / 2}\right)$ dissociation channels, the differences between appearance times, $\Delta \tau$, and the I/I* branching ratios obtained with the 9D (full dimension) surface-hopping trajectory method on the potential energy surfaces by Xie et al. [24] and the transition dipole moments of Ref. [12] are summarized in Table III and depicted in Figure 8 for the excitation wavelengths $243 \mathrm{~nm}$ and $268 \mathrm{~nm}$.

It is apparent from the semiclassical appearance times shown in the bottom panel of Figure 8 and in Table III that at both excitation wavelengths, the times associated with the $\mathrm{I}\left({ }^{2} P_{3 / 2}\right)$ and $\mathrm{I}^{*}\left({ }^{2} P_{1 / 2}\right)$ channels increase monotonically and smoothly with increasing vibrational excitation of the methyl fragment, as it was the case in the $4 \mathrm{D}$ wavepacket calculations. In the present case, however, the increase is milder and, overall, the appearance times are always smaller (by about 7-8\%) than those obtained in the 4D wavepacket calculations, in spite of using the same final C-I distance to calculate the reaction times. There must be at least one explanation for this trend, which should be related with the different dimensionality of the two models; the fact that the semiclassical calculations are full dimension (9D) implies a larger ramdomization of the available energy into all the vibrational modes of the $\mathrm{CH}_{3}$ fragment, and thus the effect of the available energy in each case is milder. This monotonous and smooth dependence of the surface hopping trajectory appearance times as the vibrational energy content of the methyl fragment increases is, also in this case, at variance with the experimental observations. Despite the difference in the dimensionality, the overall agreement between the appearance times calculated with the 4D wavepacket 
TABLE III: 9D surface hopping trajectory appearance times $\tau$ (in fs), differences between appearance times $\Delta \tau$ and $\mathrm{I} / \mathrm{I}^{*}$ branching ratios measured for the different channels of the $A$-band photodisociation of $\mathrm{CH}_{3} \mathrm{I}$ at the excitation wavelengths $\lambda_{\text {exc }} 268 \mathrm{~nm}$ and $243 \mathrm{~nm}$.

\begin{tabular}{|c|c|c|c|c|c|c|c|}
\hline \multicolumn{8}{|c|}{$\lambda_{e x c}=268 \mathrm{~nm}$} \\
\hline & $\mathrm{CH}_{3}\left(\nu_{1}, \nu_{2}\right)$ & $(0,0)$ & $(1,0)$ & $(0,1)$ & $(1,1)$ & $(0,2)$ & $(1,2)$ \\
\hline $\mathrm{I}\left({ }^{2} P_{3 / 2}\right), \tau(\mathrm{fs})$ & & 88.9 & 89.2 & 90.7 & 91.8 & 91.9 & 93.0 \\
\hline $\mathrm{I}^{*}\left({ }^{2} P_{1 / 2}\right), \tau(\mathrm{fs})$ & & 108.2 & 108.5 & 108.9 & 109.0 & 109.3 & 109.0 \\
\hline$\Delta \tau(\mathrm{fs})$ & & 19.5 & 19.3 & 18.2 & 17.2 & 17.4 & 16.0 \\
\hline $\mathrm{I} / \mathrm{I}^{*}$ ratio & & 0.09 & 0.09 & 0.28 & 0.42 & 0.57 & 1.00 \\
\hline \multicolumn{8}{|c|}{$\lambda_{e x c}=243 \mathrm{~nm}$} \\
\hline & $\mathrm{CH}_{3}\left(\nu_{1}, \nu_{2}\right)$ & $(0,0)$ & $(1,0)$ & $(0,1)$ & $(1,1)$ & $(0,2)$ & $(1,2)$ \\
\hline $\mathrm{I}\left({ }^{2} P_{3 / 2}\right), \tau(\mathrm{fs})$ & & 82.6 & 83.0 & 83.6 & 83.9 & 84.0 & 84.6 \\
\hline $\mathrm{I}^{*}\left({ }^{2} P_{1 / 2}\right), \tau(\mathrm{fs})$ & & 96.6 & 97.8 & 98.1 & 98.8 & 98.6 & 98.9 \\
\hline$\Delta \tau(\mathrm{fs})$ & & 14.0 & 14.8 & 14.5 & 14.9 & 14.6 & 14.2 \\
\hline $\mathrm{I} / \mathrm{I}^{*}$ ratio & & 1.01 & 0.79 & 1.65 & 1.46 & 1.95 & 1.79 \\
\hline
\end{tabular}

and 9D trajectory surface-hopping approaches is quite good, confirming that the degrees of freedom neglected in the quantum simulations are not really determinant to describe the photodissociation dynamics. We conclude then that the role of the conical intersection is minor, even when considering a full dimension calculation as in the present case.

The other observables, $\Delta \tau, \mathrm{I} / \mathrm{I}^{*}$ branching ratios and the umbrella mode $\nu_{2}$ vibrational distributions for the channels with $\mathrm{CH}_{3}$ in $\nu_{1}=0$ and $\nu_{1}=1$ and I and $\mathrm{I}^{*}$ (not shown), at the two excitation wavelengths $243 \mathrm{~nm}$ and $268 \mathrm{~nm}$, follow similar trends as those observed in the $4 \mathrm{D}$ wavepacket calculations and they will not be further discussed here.

\section{Ab initio potential energy of the Rydberg states of $\mathrm{CH}_{3}$}

The clocking of the photodissociation of $\mathrm{CH}_{3} \mathrm{I}$ after excitation at $268 \mathrm{~nm}$ and $243 \mathrm{~nm}$ studied in this work is carried out by probing the free $\mathrm{CH}_{3}$ fragment by resonance enhanced multiphoton ionization (REMPI) in a $2+1$ process. This means that 2 photons of the 
same color, for instance, $333.5 \mathrm{~nm}$ for the REMPI process of vibrationless $\mathrm{CH}_{3}(\nu=0)$, are absorbed simultaneously from the vibrational ground state of the electronic ground state of $\mathrm{CH}_{3}$ to the $3 p_{z}$ Rydberg electronically excited state in its vibrational ground state $\left(0_{0}^{0}\right.$ transition in the REMPI spectrum). The third photon of the same color is absorbed then from that vibrational state of the Rydberg state to the ionization continuum providing the $\mathrm{CH}_{3}^{+}$which is detected. In order to clock the reaction, the resonant condition must be fulfilled, i.e. for the spectroscopic transition to occur, the $\mathrm{CH}_{3}$ radical has to be considered free of any measurable interaction with respect to the departing iodine atom, both in the ground electronic ${ }^{2} P_{3 / 2}$ and spin-orbit excited ${ }^{2} P_{1 / 2}$ states. In addition, the substantial bandwidth of the femtosecond laser probing pulse (about $2 \mathrm{~nm}$ ) has to be considered to know when the pure spectroscopic transition occurs. The question would be then at what precise distance between the iodine and the methyl fragments, the resonant condition will be fulfilled.

To answer this question, we have carried out high level ab initio calculations of the potential energy dependence of the Rydberg states of $\mathrm{CH}_{3}$ on the $\mathrm{C}$-I distance of the dissociating $\mathrm{CH}_{3} \mathrm{I}$ molecule. Figure 10 depicts the potential energy of the different electronic states of the $\mathrm{CH}_{3}$ radical as a function of the $\mathrm{C}-\mathrm{I}$ distance considering the two asymptotes in each case, i.e. in correlation with I and I*. The Rydberg states calculated for $\mathrm{CH}_{3}$ are the $3 s$, the degenerate $3 p_{x}$ and $3 p_{y}$ and the $3 p_{z}$, which are the lowest lying electronic excited states. In addition, the ion-pair $\mathrm{I}^{-} \mathrm{CH}_{3}^{+}$state appears and interacts with the $3 s$ state at about $7 \AA$. A close inspection of the potential energy curves indicates that at shorter C-I distances than about 13 bohr (6.88 $\AA$ ), the Rydberg states start to distort (increasing in energy) and, in some cases, they split into different curves. Indeed, this is the C-I distance we have chosen to calculate the appearance times using both theoretical methods (see above).

In addition to the potential energy curves, we have calculated the corresponding adiabatic potential energy curves (adiabats) for the umbrella mode $\nu_{2}$ vibrational states of $\mathrm{CH}_{3}$. One possible explanation for the non-monotonous trend in the observed clocking times (see Figure 7) could be that the C-I distance, where the distortion of the potential energy starts given the proximity of the heavy iodine atom, would be different for the different $\nu_{2}$ vibrational states of $\mathrm{CH}_{3}$ and, thus, each pure vibronic transition $\left(0_{0}^{0}, 1_{1}^{1}, 2_{1}^{1}\right.$ and $\left.2_{2}^{2}\right)$ providing the clocking of each channel would occur at a slightly different C-I distances. Figure 11 depicts the potential energy difference between the adiabats of the electronic ground state of $\mathrm{CH}_{3}$ 


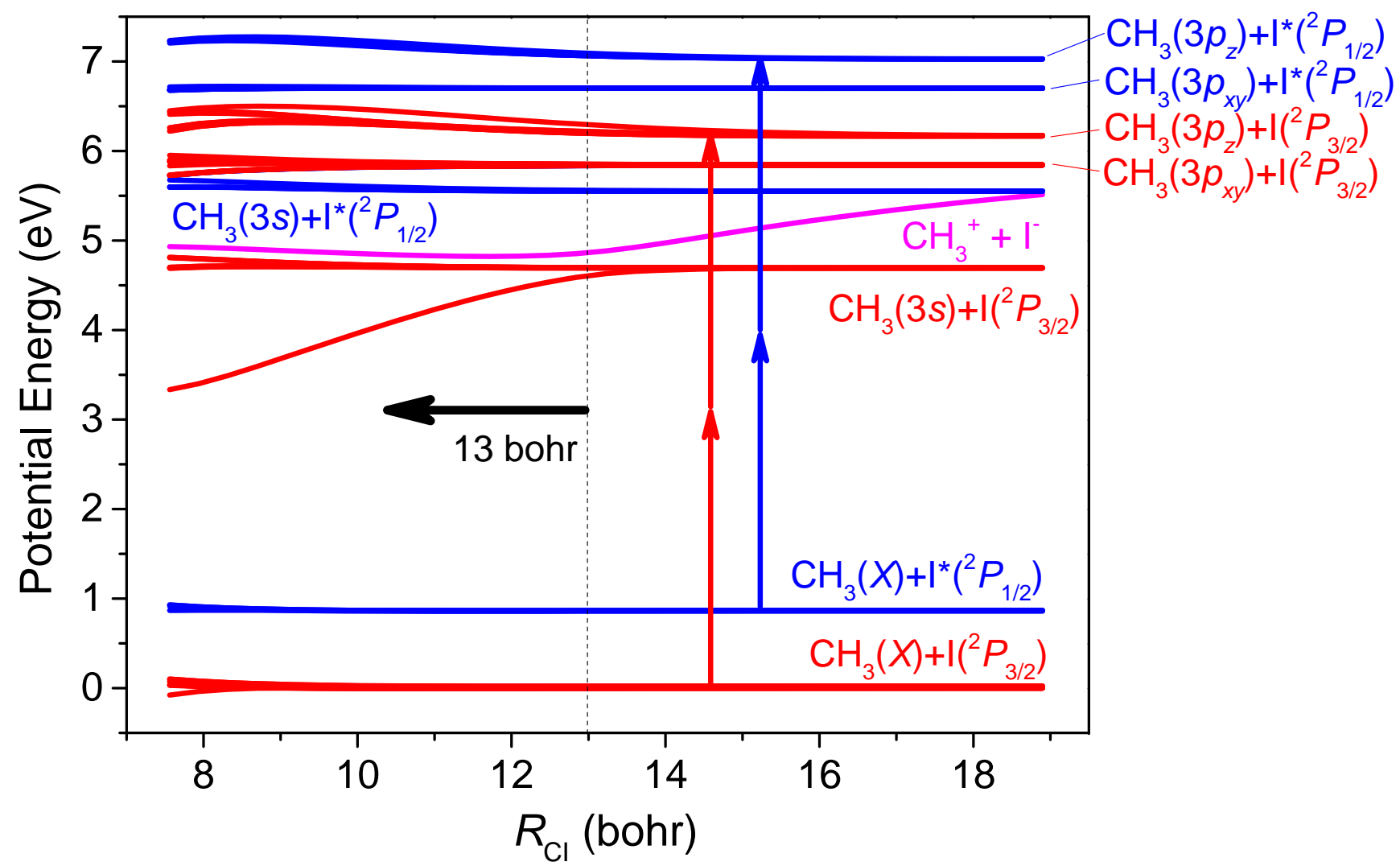

FIG. 10: Potential energy as a function of the C-I distance for the different electronic states of the $\mathrm{CH}_{3}$ radical, including the electronic ground state, the $3 s, 3 p_{x y}$ and the $3 p_{z}$ Rydberg states, in correlation with I and $\mathrm{I}^{*}$, and the ion-pair $\mathrm{I}^{-} \mathrm{CH}_{3}^{+}$state, calculated at the $a b$ initio level described in Section II.D. The dashed vertical line represents the C-I distance below of which the potential energy of the Rydberg states start to change (increase) as the iodine atom is approaching to the $\mathrm{CH}_{3}$ fragment. This is the C-I distance (13 bohr) chosen in the theoretical treatments to calculate the appearance times. The double arrows (red and blue) represent the initial two-photon absorption for the $2+1$ REMPI process employed for fragment detection.

and those of the $3 p_{z}$ Rydberg state, which are involved in the $2+1$ REMPI process used for detection of the different vibrational states of the free $\mathrm{CH}_{3}$. As can be seen, all the differences between the adiabat curves are rather similar and parallel and, thus, the vibronic transitions involved in the clocking of the different vibrational states of $\mathrm{CH}_{3}$ would occur at approximately the same C-I distance of the photodissociating molecule. The conclusion 
is that the probing step would not explain neither the non-monotonous appearance times measured for the different vibrational states of the $\mathrm{CH}_{3}$ fragment.

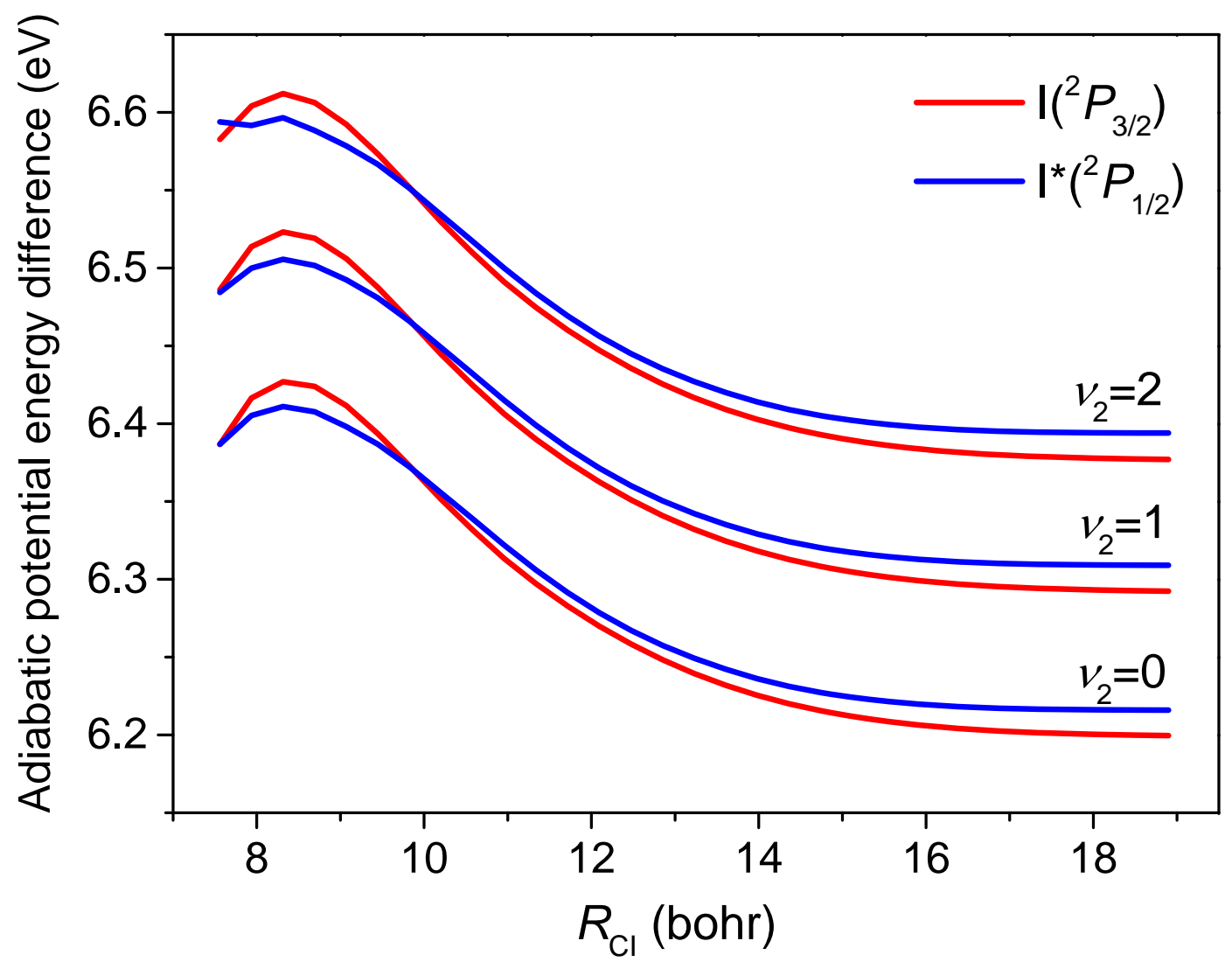

FIG. 11: Differences between the ground state and $3 p_{z}$ Rydberg adiabatic potential energy curves of $\mathrm{CH}_{3}$ as a function of the C-I distance, calculated at the ab initio level described in Section II.D.

\section{DISCUSSION}

One of the issues that has provoked controversy around the photodissociation of $\mathrm{CH}_{3} \mathrm{I}$ in the $A$-band is the relative contribution to absorption to different excited states across the band. It is well established that in the center of the band at around $260 \mathrm{~nm}$, absorption to the ${ }^{3} Q_{0}$ state is predominant, adiabatically leading to the formation of spin-orbit excited $I^{*}\left({ }^{2} P_{1 / 2}\right)$. Since the strength of absorption to the ${ }^{1} Q_{1}$ state is very low, the observed ground state iodine $\mathrm{I}\left({ }^{2} P_{3 / 2}\right)$ must originate mostly non-adiabatically from the existing ${ }^{3} Q_{0} /{ }^{1} Q_{1}$ 
conical intersection. The issue was addressed in previous experimental work [13], which concluded that the participation of the ${ }^{1} Q_{1}$ state is dominant only at the blue edge of the absorption band at $217 \mathrm{~nm}$, but very significantly reduced already at $230 \mathrm{~nm}$, as it is also deduced from the $\beta_{2}$ anisotropy parameters depicted in Figure 3. For the absorption examined in this work at the blue side of the absorption band at $243 \mathrm{~nm}$, the strong parallel character observed in the methyl images correlating with both I/I* channels and the large values of the $\beta_{2}$ anisotropy parameter close to the maximum of 2 for a parallel transition, constitutes further indication of the minor role played by the ${ }^{1} Q_{1}$ state at this excitation wavelength. However, we have observed longer reaction times at $\lambda_{e x c}=243 \mathrm{~nm}$ when excitation to the umbrella mode of $\mathrm{CH}_{3}$ is produced in comparison with vibrationless $\mathrm{CH}_{3}$ for both $\nu_{1}=0$ and $\nu_{1}=1$ and for both electronic channels yielding I and $I^{*}$ (see Table and Figure). This trend is surprising and unexpected when compared to the results obtained at $\lambda_{\text {exc }}=268 \mathrm{~nm}$ and cannot be attributed to the probing step, which is exactly the same for the experiments carried out at the two excitation wavelengths.

Interestingly, Warne et al. [33] reported recently experiments carried out at $269 \mathrm{~nm}$ and $255 \mathrm{~nm}$ using 2-photon ionization probe at $395 \mathrm{~nm}$ in time-resolved photoelectron imaging, in which they observe different dynamics at the two excitation wavelengths with significant changes in the lifetimes and dynamic structure. The lifetime measured for the departure of the initial wavepacket out of the ionization Franck-Condon region after excitation at 268 $\mathrm{nm}$ was found to be $30 \mathrm{fs}$, which would be consistent with measurements of the formation of products carried out in the present work. However, after excitation at $255 \mathrm{~nm}$ although a similar process is observed in a first decay component, a secondary feature associated with a delayed component by about $100 \mathrm{fs}$ is found. The authors of Ref. [33] suggest that this delayed feature is due to excited state population and specific dynamics on the ${ }^{1} Q_{1}$ potential energy surface, undergoing significant rearrangement prior to dissociation. This explanation is, however, not very plausible in view of the 4D wavepacket and 9D surface-hopping trajectory calculations carried out in this work, which demonstrate that an absorption spectrum where the contribution of the ${ }^{1} Q_{1}$ state is overestimated, cannot explain a distinct dynamics occurring in this state (see, for instance, Figure 8). It may happen that the accidental resonance in the probing step, where resonance enhanced ionization through the $6 p$ Rydberg state occurs, can explain the distinct features found at this excitation wavelength. More experiments of the same class but carried out at different probing wavelengths to avoid any 
accidental resonance may help to understand the observed features.

Once the role of the absorption to the ${ }^{1} Q_{1}$ state or the ${ }^{3} Q_{0} /{ }^{1} Q_{1}$ conical intersection can be disregarded as a cause of the characteristic appearance times observed as a function of the final umbrella vibrational quantum state of $\mathrm{CH}_{3}$ at the two excitation wavelengths studied in this work (268 and $243 \mathrm{~nm}$ ), we have to rely again on the $(2+1)$ REMPI probing step through the $3 p_{z}$ Rydberg state of $\mathrm{CH}_{3}$ to try to explain the observations. We have demonstrated in the previous section that $a b$ initio calculations of the adiabatic potential energy curves (as a function of the C-I distance) cannot explain that the optical window determined by the bandwidth of the probe laser pulse accessed by two-photon absorption is open at different C-I distances for the different umbrella vibrational states of $\mathrm{CH}_{3}$ (see Figures 10 and 11), which might explain the experimental results. However, it may happen that the efficiency of the two-photon absorption at the specific wavelengths for the $0_{0}^{0}, 2_{1}^{1}$ and $2_{2}^{2}$ transitions in $\mathrm{CH}_{3}$ depends on the final vibrational state of the $3 p_{z}$ Rydberg state. It is well known, that the $3 p_{z}$ Rydberg state of $\mathrm{CH}_{3}$ is predissociative due to the presence of valence states [5860]. The predissociation rate depends on the vibrational state accessed in the $3 p_{z}$ Rydberg state of the fragment, being faster as vibrational excitation increases [58]. Actually, REMPI spectroscopy of $\mathrm{CH}_{3}$ is limited by the extension of this predissociation, which precludes detection of highly excited vibrationally states. We do not know the effect of the presence of the heavy iodine atom on the valence states and on the predissociation rate, but if higher vibrational states of the Rydberg would be more affected by the presence of the iodine atom, then unexpected effects on the probing process could arise, affecting the appearance times in an undetermined way. Clocking experiments on the isotopic variant $\mathrm{CD}_{3} \mathrm{I}$ would be very timely as predissociation is less effective in deuterated methyl $\left(\mathrm{CD}_{3}\right)$. Moreover, given that the Franck-Condon region accessed by excitation at $243 \mathrm{~nm}$ is somewhat different than that at $268 \mathrm{~nm}$ excitation, the combination of the $243 \mathrm{~nm}$ pump and (2+1) REMPI detection of the $\mathrm{CH}_{3}$ fragment can yield different dependence on the clocking times of the umbrella vibrational mode excitation than at $268 \mathrm{~nm}$.

Unfortunately, none of the probe spectroscopies used recently to study the time-resolved photodissociation of $\mathrm{CH}_{3} \mathrm{I}$ in the $A$-band, which includes two-photon ionization photoelectron spectroscopy, soft X-ray inner-shell photoelectron spectroscopy, VUV single-photon ionization and XUV core-to-valence transient absorption spectroscopy, have been able of measuring vibrationally state-resolved lifetimes or appearance times. The use of other laser 
spectroscopic techniques for detection of quantum state-resolved $\mathrm{CH}_{3}$ without the limitations of REMPI would be very timely to confirm or disregard the vibrational state effects on the clocking times.

\section{CONCLUSIONS}

A critical report on the clocking of the chemical bond cleavage at the heart of Femtochemistry has been presented by experimentally and theoretically studying the photodissociation of $\mathrm{CH}_{3} \mathrm{I}$ upon absorption in the center $(268 \mathrm{~nm})$ and in the blue side $(243 \mathrm{~nm})$ of the $A$-band. The clocking of vibrationally and electronic state-resolved channels of the reaction is carried out by resonance enhanced multiphoton ionization of the $\mathrm{CH}_{3}$ fragment. In particular, vibrationless methyl and vibrationally excited to the $\nu_{2}$ umbrella bending and $\nu_{1}$ symmetric stretch modes have been detected by $(2+1)$ REMPI through the intermediate $3 p_{z}$ Rydberg state. Distinct trends of the clocking times as a function of the vibrational excitation of $\mathrm{CH}_{3}$ are observed at both excitation wavelengths, which are at variance of theoretical calculations consisting in 4D wavepacket and 9D trajectory surface hopping carried out on the available full dimension ab initio potential energy surfaces and transition dipole moments for the ground and excited ${ }^{3} Q_{0}$ and ${ }^{1} Q_{1}$ states. The role of the ${ }^{3} Q_{0} /{ }^{1} Q_{1}$ conical intersection or a significant absorption to the ${ }^{1} Q_{1}$ state have been discarded to explain the observed vibrationally state-resolved clocking times at the two excitation wavelengths. Ab initio calculations of the adiabatic ground and excited state potential energy curves of the $\mathrm{CH}_{3}$ radical as a function of the distance to the heavy iodine atom cannot account for specific opening of the optical window for different vibrational states of $\mathrm{CH}_{3}$. However, the non-adiabatic coupling between valence states and the $3 p_{z}$ Rydberg state of the $\mathrm{CH}_{3}$ fragment gives rise to

efficient electronic predissociation whose rate increases with the vibrational excitation. The presence of the heavy iodine atom may affect differently to the couplings and predissociation rate of the different vibrational states of $\mathrm{CH}_{3}$ and this would have unpredictable effects on the clocking times measured. More experiments using deuterated $\mathrm{CD}_{3} \mathrm{I}$, where $\mathrm{CD}_{3}$ predissociation is much less efficient, and/or the use of a different laser spectroscopy technique, which allows us detection of quantum state-resolved products, would be important to finally disentangle the effect of internal degrees of freedom of the molecular fragment in the clocking of state-resolved channels in the photodissociation of $\mathrm{CH}_{3} \mathrm{I}$. 


\section{ACKNOWLEDGMENT}

M. L. M.-S. acknowledges financial support through a predoctoral contract from Universidad Complutense de Madrid. M. E. C. is grateful to the Spanish MINECO for a contract through Programa de Técnicos de Apoyo a Infraestructuras. This work has been financed by the Spanish MINECO and MICIU (grants CTQ2016-75880-P, FIS2016-77889-R and PGC2018-096444-B-I00). This research has been carried out within the Unidad Asociada Química Física Molecular between the Departamento de Química Física of Universidad Complutense de Madrid and CSIC.The facilities provided by the Center for Ultrasfast Lasers of Universidad Complutense de Madrid are acknowledged.

[1] M. J. Rosker, M. Dantus, and A. H. Zewail, J. Chem. Phys. 89, 6113 (1988).

[2] A. H. Zewail, Science 242, 1645 (1988).

[3] A. H. Zewail, Angew. Chem., Int. Ed. 39, 2586 (2000).

[4] M. Dantus, M. J. Rosker, and A. H. Zewail, J. Chem. Phys. 89, 6128 (1988).

[5] T. S. Rose, M. J. Rosker, and A. H. Zewail, J. Chem. Phys. 91, 7415 (1989).

[6] A. H. Zewail, J. Phys. Chem. A 102, 4031 (1998).

[7] A. Gedanken and M. D. Rowe, Chem. Phys. Lett. 34, 39 (1975).

[8] Y. Amatatsu, S. Yabushita, and K. Morokuma, J. Chem. Phys. 104, 9783 (1996).

[9] D. W. Chandler and P. L. Houston, J. Chem. Phys. 87, 1445 (1987).

[10] A. T. J. B. Eppink and D. H. Parker, J. Chem. Phys. 109, 4758 (1998).

[11] A. T. J. B. Eppink and D. H. Parker, J. Chem. Phys. 110, 832 (1999).

[12] L. Rubio-Lago, A. García-Vela, A. Arregui, G. A. Amaral, and L. Bañares, J. Chem. Phys. 131, 174309 (2009).

[13] M. G. González, J. Rodríguez, L. Rubio-Lago, A. García-Vela, A. Arregui, G. A. Amaral, and L. Bañares, Phys. Chem. Chem. Phys. 13, 16404 (2011).

[14] R. de Nalda, J. G. Izquierdo, J. Durá, and L. Bañares, J. Chem. Phys. 126, 021101 (2007).

[15] R. de Nalda, J. Durá, A. García-Vela, J. G. Izquierdo, González-Vázquez, and L. Bañares, J. Chem. Phys. 128, 244309 (2008).

[16] A. García-Vela, R. de Nalda, J. Durá, J. González-Vázquez, and L. Bañares, J. Chem. Phys. 
135, 154306 (2011).

[17] M. E. Corrales, V. Loriot, G. Balerdi, J. González-Vázquez, R. de Nalda, L. Bañares, and A. H. Zewail, Phys. Chem. Chem. Phys. 16, 8812 (2014).

[18] J. Durá, R. de Nalda, J. Álvarez, J. G. Izquierdo, G. A. Amaral, and L. Bañares, ChemPhysChem 9, 1245 (2008).

[19] J. Durá, R. de Nalda, G. A. Amaral, and L. Bañares, J. Chem. Phys. 131, 14 (2009).

[20] Y. Amatatsu, K. Morokuma, and S. Yabushita, J. Chem. Phys. 94, 4858 (1991).

[21] H. Guo and G. C. Schatz, J. Chem. Phys. 93, 393 (1990).

[22] H. Guo, J. Chem. Phys. 96, 6629 (1992).

[23] A. D. Hammerich, U. Manthe, R. Kosloff, H.-D. Meyer, and L. S. Cederbaum, J. Chem. Phys. 101, 5623 (1994).

[24] D. Q. Xie, H. Guo, Y. Amatatsu, and R. Kosloff, J. Phys. Chem. A 104, 1009 (2000).

[25] A. B. Alekseyev, H. Liebermann, R. J. Buenker, and S. N. Yurchenko, J. Chem. Phys. 126, $234102(2007)$.

[26] A. B. Alekseyev, H. Liebermann, and R. J. Buenker, J. Chem. Phys. 126, 234103 (2007).

[27] C. R. Evenhuis and U. Manthe, J. Phys. Chem. A 115, 5992 (2011).

[28] N. Wittenbrink and W. Eisfeld, J. Chem. Phys. 148, 094102 (2018).

[29] A. R. Attar, A. Bhattacherjee, and S. R. Leone, J. Phys. Chem. Lett. 6, 5072 (2015).

[30] L. Drescher, M. C. E. Galbraith, G. Reitsma, J. Durá, N. Zhavoronkov, S. Patchkovskii, M. J. J. Vrakking, and J. Mikosch, J. Chem. Phys. 145, 011101 (2016).

[31] A. Baumann, D. Rompotis, O. Schepp, and M. Wieland, J. Phys. Chem. A 122, 4779 (2018).

[32] F. Brauße, G. Goldsztejn, K. Amini, R. Boll, S. Bari, C. Bomme, M. Brouard, M. Burt, B. C. de Miranda, S. D'usterer, et al., Phys. Rev. A 97, 043429 (2018).

[33] E. M. Warne, B. Downes-Ward, J. Woodhouse, M. A. Parkes, D. Bellshaw, E. Springate, P. Majchrzak, Y. Zhang, G. Karras, A. S. Wyatt, et al., Phys. Chem. Chem. Phys. 21, 11142 (2019).

[34] A. T. Eppink and D. H. Parker, Rev. Sci. Instrum. 68, 3477 (1997).

[35] G. A. Garcia, L. Nahon, and I. Powis, Rev. Sci. Instrum. 75, 4989 (2004).

[36] V. Loriot, R. de Nalda, and L. Bañares, App. Sci. 8, 1227 (2018).

[37] W. Arbelo-González, L. Bonnet, and A. García-Vela, Phys. Chem. Chem. Phys. 15, 9994 (2013). 
[38] W. Arbelo-González, L. Bonnet, and A. García-Vela, J. Chem. Phys. 142, 134111 (2015).

[39] M. Richter, P. Marquetand, J. González-Vázquez, I. Solá, and L. González, Journal of Chemical Theory and Computation 7, 1253 (2011).

[40] H.-J. Werner, P. J. Knowles, R. Lindh, F. R. Manby, M. Schütz, P. Celani, T. Korona, A. Mitrushenkov, G. Rauhut, T. B. Adler, et al., MOLPRO, version 2012, a package of ab initio programs.

[41] J. Petersen and R. Mitrić, Physical Chemistry Chemical Physics 14, 8299 (2012), ISSN 14639084 .

[42] J. C. Corchado and J. Espinosa-García, Phys. Chem. Chem. Phys. 11, 10157 (2009).

[43] C. W. Eaker and G. C. Schatz, J. Chem. Phys. 81, 2394 (1984).

[44] C. W. Eaker, G. C. Schatz, N. DeLeon, and E. J. Heller, J. Chem. Phys. 81, 5913 (1984).

[45] J. Binney and D. Spergel, Monthly Roy. Astron. Soc 206, 159 (1964).

[46] C. C. Martens and G. S. Ezra, J. Chem. Phys. 83, 2990 (1985).

[47] L. J. Curtis and D. G. Ellis, Am. J. Phys. 72, 1521 (2004).

[48] S. A. Vázquez and E. Martínez-Núñez, Phys. Chem. Chem. Phys. 17, 6948 (2015).

[49] M. J. Wilhelm, E. Martínez-Núñez, J. González-Vázquez, S. A. Vázquez, J. M. Smith, and H.-L. Dai, ApJ 15, 849 (2017).

[50] B. O. Roos, R. Lindh, P.-A. Malmqvist, V. Veryazov, and P.-O. Widmark, The Journal of Physical Chemistry A 108, 2851 (2004), URL http://pubs.acs.org/doi/abs/10.1021/ jp031064+.

[51] M. Reiher and A. Wolf, The Journal of chemical physics 121, 10945 (2004), URL http: //scitation.aip.org/content/aip/journal/jcp/121/22/10.1063/1.1818681.

[52] B. Schimmelpfennig, Stockholm University (1996).

[53] I. Fernández Galván, M. Vacher, A. Alavi, C. Angeli, F. Aquilante, J. Autschbach, J. J. Bao, S. I. Bokarev, N. A. Bogdanov, R. K. Carlson, et al., Journal of Chemical Theory and Computation (2019), ISSN 1549-9618, 00001.

[54] C. C. Martson and G. G. Balint-Kurti, J. Chem. Phys. 91, 3571 (1989).

[55] G. Gitzinger, M. E. Corrales, V. Loriot, R. de Nalda, and L. Bañares, J. Chem. Phys. 136, 074303 (2012).

[56] A. García-Vela and L. Bañares, Phys. Chem. Chem. Phys. 13, 2228 (2011).

[57] A. García-Vela and L. Bañares, Eur. Phys. J. D 67, 265 (2013). 
[58] G. Balerdi, J. Woodhouse, A. Zanchet, R. de Nalda, M. L. Senent, García-Vela, and L. Bañares, Phys. Chem. Chem. Phys. 18, 110 (2016).

[59] S. Marggi Poullain, D. V. Chicharro, A. Zanchet, M. G. González, L. Rubio-Lago, M. L. Senent, García-Vela, and L. Bañares, Phys. Chem. Chem. Phys. 18, 17054 (2016).

[60] A. Zanchet, L. Bañares, M. L. Senent, and García-Vela, Phys. Chem. Chem. Phys. 18, 33195 (2016). 